Bundesgesundheitsbl 2016 · 59:662-678

DOI 10.1007/s00103-016-2342-7

Online publiziert: 19. April 2016

(C) The Author(s) 2016. This article is available at SpringerLink with Open Access

CrossMark

Ulrike Dapp ${ }^{1} \cdot$ Martin Dirksen-Fischer ${ }^{2}$. Gudrun Rieger-Ndakorerwa ${ }^{3}$. Regina Fertmann ${ }^{4} \cdot$ Klaus-Peter Stender ${ }^{4} \cdot$ Stefan Golgert ${ }^{1} \cdot$ Wolfgang von Renteln-Kruse ${ }^{1} \cdot$ Christoph E. Minder ${ }^{5}$

'Albertinen-Haus Zentrum für Geriatrie und Gerontologie, Wissenschaftliche Einrichtung an der Universität Hamburg, Hamburg, Deutschland

${ }^{2}$ Institut für Hygiene und Umwelt, Freie und Hansestadt Hamburg, Behörde für Gesundheit und Verbraucherschutz, Hamburg, Deutschland

${ }^{3}$ Fachamt Gesundheit, Freie und Hansestadt Hamburg, Bezirksamt Eimsbüttel, Hamburg, Deutschland ${ }^{4}$ Fachabteilung Gesundheitsdaten und Gesundheitsförderung, Freie und Hansestadt Hamburg, Behörde für Gesundheit und Verbraucherschutz, Hamburg, Deutschland

${ }^{5}$ Horten-Zentrum, Universitätsspital Zürich, Zürich, Schweiz

\title{
Vergleichbarkeit von Studien epidemiologischer Alternsforschung
}

\section{Ergebnisse aus der Longitudinalen Urbanen Cohorten-Alters-Studie (LUCAS) und drei repräsentativen Hamburger Querschnitt- Studien zur Gesundheit im Alter}

naler Kompetenz mit der Folge von Hilfsund Pflegebedürftigkeit sowie Tod [4]. Ursächlich werden verringerte physiologische Reservekapazitäten verschiedener Körpersysteme angenommen (z. B. Immunsystem, zentrales Nervensystem, Muskulatur). Ein fortschreitender Verlust von Skelettmuskelmasse und muskulärer Kraft (Sarkopenie) wird als eine der Schlüsselkomponenten angesehen. Ernährungsfaktoren und körperliche Aktivität sind modulierende Einflüsse. Zur Operationalisierung von Frailty dienen zwei Ansätze, das sog. Phänotypmodell [5] und das Defizitmodell [6]. Psychosoziale Faktoren sind bislang im Frailty-Konzept kaum berücksichtigt [7].

Bevölkerungsmedizinisch ist die ausgeprägte, klinisch manifeste Frailty (Gebrechlichkeit) die Form der Alterung, die mit dem Höchstmaß an Versorgungsproblemen verknüpft ist. Deshalb stellt sich die zentrale Frage nach der positiven Beeinflussbarkeit [4], und nicht zuletzt begründen die resultierenden finanziellen
Belastungen die wachsende Bedeutung von Gesundheitsförderung sowie proaktiver Maßnahmen auch für älter werdende Menschen [8]. Bevölkerungsbasierte Langzeitkohortenstudien sind nützlich, um komplexe Interaktionen zwischen körperlichen, psychischen und sozialen Faktoren zu untersuchen [1]. Mit steigender Lebenserwartung nehmen die Jahre in Gesundheit zu, ebenso verschiebt sich der Anteil an Jahren in beeinträchtigter funktionaler Gesundheit zunehmend ins höhere Lebensalter $[9,10]$. Insbesondere hochaltrige Menschen werden jedoch häufig aus epidemiologischen Studien ausgeschlossen (z. B. durch eine obere Altersgrenze), wodurch die Nutzung von Studienergebnissen bezüglich der eingangs genannten Herausforderungen reduziert ist [11].

Die Longitudinale Urbane Cohorten-Alters-Studie (LUCAS) wurde in einem interdisziplinären Prozess entwickelt, um individuelle Alternsverläufe von über 3300 60-jährigen und älteren, selbstständig und initial ohne Pflegestufe 


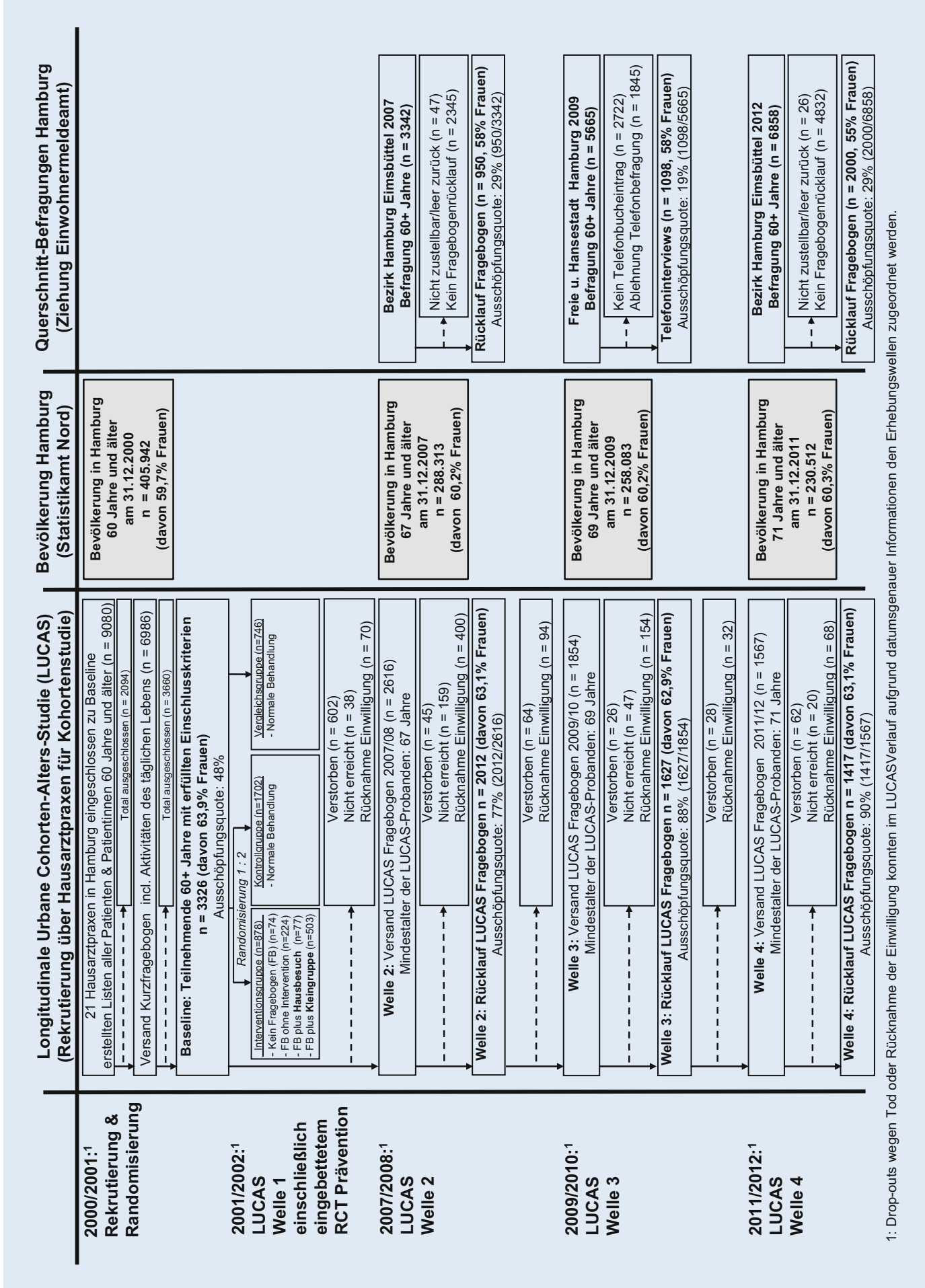

Abb. $1 \triangleleft$ Zeitliche Übersicht der ersten vier LUCAS-Wellen 1-4 sowie der Datenquellen des Statistikamtes Nord und der drei Querschnittbefragungen zur Gesundheit im Alter in Hamburg inkl. Stichprobengrößen und Ausschöpfung

lebenden Menschen in einer urbanen Umgebung über bisher 15 Jahre zu erfassen. Teilnehmende Personen wurden in bisher sechs Wellen zu gesundheitsbezogenen Merkmalen umfassend befragt. Die gestellten Fragen stammen aus der Praxis von Gesundheitsförderung und Prävention für selbstständig lebende ältere Menschen sowie auch aus Versorgungsbereichen für die betagte Bevölkerung [12]. Ziel ist (a) die Generierung präklinischer Marker sich entwickelnder Verluste funktionaler Kompetenz [13], (b) die Validierung von Verfahren zur Erkennung verschiedener Stufen/Grade von Mobilität, Sturzrisiko und Frailty
[14] sowie (c) die Analyse nachhaltiger Effekte gesundheitsfördernder Interventionen in randomisierten kontrollierten Studien (RCT), die im Studiendesign eingebettet wurden [15]. Zu Studienbeginn im Jahr 2000 wurde deshalb entschieden, ältere Menschen über Hausarztpraxen zu rekrutieren. Im Unterschied zu anderen 
Bundesgesundheitsbl 2016 59:662-678 DOI 10.1007/s00103-016-2342-7

(c) The Author(s) 2016

U. Dapp · M. Dirksen-Fischer · G. Rieger-Ndakorerwa $\cdot$ R. Fertmann · K.-P. Stender · S. Golgert · W. von Renteln-Kruse · C. E. Minder

\section{Vergleichbarkeit von Studien epidemiologischer Alternsforschung. Ergebnisse aus der Longitudinalen Urbanen Cohorten-Alters-Studie (LUCAS) und drei repräsentativen Hamburger Querschnitt-Studien zur Gesundheit im Alter}

\section{Zusammenfassung}

Hintergrund. Erhebungen mit Anspruch auf Repräsentativität erfolgen üblicherweise über eine Zufallsauswahl aus Einwohnermelderegistern. Die Teilnehmenden (60 Jahre und älter) der Langzeitkohortenstudie LUCAS wurden jedoch über Hausarztpraxen rekrutiert. Von Interesse ist die Vergleichbarkeit, da die LUCAS-Datenbank umfangreiche Gesundheitsinformationen zum Altern enthält.

Ziel der Arbeit. Überprüft wurde, ob Ergebnisse der während der ersten 12 Jahre erhobenen Daten der LUCAS-Kohorte vergleichbar sind mit der Bevölkerungsstatistik (Alter, Geschlecht) und mit Merkmalen (Soziodemografie, Gesundheit) von drei Befragungen mit repräsentativer Stichprobenziehung über das Einwohnermelderegister Hamburg.
Methoden. Alters- und geschlechtsspezifische Angaben des Statistikamtes wurden aufbereitet, um diese mit denen der LUCASKohorte für die Rekrutierung (2000/01) und Wellen zwei bis vier zu vergleichen. Außerdem wurden identisch erfasste Merkmale der drei Querschnittbefragungen mit denen der zeitnahen LUCAS-Wellen verglichen. Ergebnisse. Der Vergleich der LUCAS-Kohorte mit den Hamburger Bevölkerungspyramiden zeigt für alle LUCAS-Wellen eine gute Übereinstimmung, ebenso der Vergleich mit Gesundheitsdaten der drei repräsentativen Querschnittbefragungen (z. B. pflegebedürftig 2012: LUCAS 7,1 \%; Hamburg 7,4 \%). Diskussion. Aus der guten Vergleichbarkeit sowohl soziodemografischer als auch gesundheitlicher Daten der LUCAS-Kohorte kann gefolgert werden, dass die in der LUCAS-Datenbank verfügbaren Angaben weitgehende Gültigkeit für die ältere Hamburger Bevölkerung haben. Da insbesondere hochaltrige Menschen in epidemiologischen Studien oft unterrepräsentiert sind, können die Ergebnisse der LUCAS-Studie für Hamburg sowie ggf. für andere deutsche Metropolregionen als aussagefähige und hilfreiche Informationsquelle dienen.

Schlüsselwörter

Selbstständig lebend · Ältere Menschen · Gesundheit · Repräsentativität . Langzeitkohortenstudie

\section{Comparability of studies of epidemiological research on aging. Results from the Longitudinal Urban Cohort Ageing Study (LUCAS) and three representative Hamburg cross-sectional studies of healthy aging}

\section{Abstract}

Background. Participants of the Longitudinal Urban Cohort Ageing Study (LUCAS) were recruited from patients 60 years and older from general practitioner's offices in Hamburg. This is different from the usual methods of drawing representative samples.

Objectives. The research question addressed the comparability of LUCAS results with those from cross-sectional surveys with participants randomly chosen from a population list. Therefore, the LUCAS data collected in four waves during the first 12 years were compared with data (age, gender) from the Hamburg Statistics Office (HSO), and selected characteristics (socio-demography, health, mobility) from three representative crosssectional surveys in older Hamburg citizens.
Methods. First, HSO data compiled in population pyramids for older men and women were compared with equivalent pyramids based on the LUCAS data at recruitment (2000/01) and in waves 2 to 4 . Second, characteristics worded identically in the cross-sectional surveys and the simultaneous LUCAS waves were compared.

Results. The LUCAS population pyramids were in good accordance at all time points with those of the general older population in Hamburg (except ages 60-64 in men in 2000). Good comparability was also found for health related characteristics from the three representative studies and simultaneous LUCAS waves (e. g. need of nursing care in 2012: LUCAS $7.1 \%$; Hamburg $7.4 \%$ ).
Conclusions. Information on health in old age generated periodically in the LUCAS cohort was largely comparable with that from representative cross-sectional studies and statistics registries. Older people are frequently under-represented in epidemiological studies. Therefore, the LUCAS data may provide useful information for Hamburg and similar metropolitan areas in Germany.

Keywords Independant living · Aged citizens · Health . Representativeness · Longitudinal cohort study
Datenquellen mit Stichprobenziehungen aus Einwohnermelderegistern [16] zielte die Repräsentativität in LUCAS eben auf diese Population (vgl. Methoden, Datenquelle 2).

Wird die Frage nach Repräsentativität einer Studie gestellt, werden meist Daten der Statistischen Landesämter bzw. des Statistisches Bundesamts (z. B. Mi- krozensus, Bevölkerungsfortschreibung) zum Vergleich der Verteilung von Merkmalen wie z. B. Alter und Geschlecht herangezogen.

Im Hinblick auf die grundlegend andere LUCAS Rekrutierungsstrategie über Hausarztpraxen werden über den Vergleich mit der amtlichen Bevölkerungsstatistik hinaus weitere Vergleiche durch- geführt. Da die Rekrutierung über Hausarztpraxen dazu führen kann, dass auch weniger gesunde Personen teilnehmen - im Unterschied zu registerbasierten Befragungen, an denen sich möglicherweise eher Gesündere beteiligen - wird die Häufigkeit verschiedener Merkmale aus drei LUCAS-Befragungswellen mit denen von drei zeitnah durchgeführten 
repräsentativen Querschnittbefragungen (Bundesland Hamburg) im selben Untersuchungsraum verglichen. Insbesondere werden soziodemografische, gesundheitliche und mobilitätsrelevante Merkmale betrachtet, die die amtliche Statistik nicht zur Verfügung stellen kann.

Die drei Querschnittbefragungen eignen sich deshalb, weil diese jeweils die 60-jährige und ältere Bevölkerung, ein Viertel der Hamburger Gesamtbevölkerung, umfassen. Eine Befragung wurde Hamburg-weit durchgeführt, die anderen im Bezirk Hamburg-Eimsbüttel mit einer Gesamtbevölkerung von fast 250.000 Einwohnern (62.550 Einwohner ab 60 Jahre). Die Größenordnung dieses Bezirks entspricht auch bezüglich der differenzierten Sozialstruktur einer deutschen Großstadt.

\section{Fragestellung}

In dieser Arbeit wird vier Fragen nachgegangen:

1. Inwiefern sind die über Hausarztpraxen rekrutierten Probanden der LUCAS-Langzeitkohorte zur Baseline und im Verlauf (Befragungswellen) bezüglich Alter und Geschlecht auf Basis der amtlichen Statistik mit der älteren Hamburger Bevölkerung vergleichbar?

2. In welchen gesundheitlich relevanten Bereichen sind die in den LUCASBefragungswellen erhobenen Daten vergleichbar mit denjenigen aus drei repräsentativen Hamburger Querschnittbefragungen?

3. Welche methodischen oder anderweitigen Erklärungen lassen sich für etwaige Abweichungen zwischen Erhebungen mit Anspruch auf Repräsentativität und Ergebnissen der LUCAS-Erhebungswellen finden?

Zusammengefasst lautet also die Hauptfragestellung:

4. Wie beeinflusst die Rekrutierungsstrategie Antworten zu gesundheitlichen Fragen in einer Erhebung via Hausarztpraxen (nicht repräsentativ bezüglich Arztpraxen, komplette Erfassung aller älteren Personen, mit niedriger Ausfallrate) gegenüber herkömmlichen Registerstudien und üblichen Probandenziehungen (repräsentative Auswahl mit eher höherer Ausfallrate und Ausschluss der Hochaltrigen)?

Nach Kenntnis der Autoren sind derartige Vergleiche bisher kein geläufiger Studiengegenstand. So ergab die Kombination der Begriffe „representativeness AND cohort" im Titel 12 Treffer in Pubmed (30.4.2015). Keine dieser Arbeiten hat Ergebnisse von Langzeitkohorten und repräsentativen Querschnittbefragungen verglichen.

Auch in Serien sukzessiver Querschnittstudien ist die Analyse individueller Verläufe der Studienteilnehmenden nicht möglich. Langzeitstudien ermöglichen hingegen die Untersuchung individueller Verläufe über die Zeit. Entwickelt sich beispielsweise bei Personen mit Frailty im Verlauf mit hoher Wahrscheinlichkeit Pflegebedürftigkeit, so liegt der Schluss nahe, dass das FrailtySyndrom wesentlich dazu beigetragen hat. Die Ergebnisse einer Studie gelten strikt nur für die untersuchte Studienpopulation. Für die Übertragbarkeit auf eine andere Population muss weitgehende Vergleichbarkeit zwischen den beiden Populationen gelten. Deshalb kommt der Vergleichbarkeit zwischen Querschnittstudien und der LUCAS-Kohorte hohe Bedeutung zu.

\section{Methoden}

Die Daten aus vier LUCAS-Befragungswellen werden hier auf Repräsentativität untersucht. Der Begriff Repräsentativität bezeichnet ein Ideal, das real nie völlig erreichbar ist (präzise definierte, statische Population ohne Wanderungen, eine perfekte Liste aller Populationsmitglieder, [d. h. keine falschen oder fehlenden Personen], mathematisch zufällige Stichprobenauswahl und komplette Teilnahme aller in die Stichprobe gewählten Populationsmitglieder [keine Nichtteilnahme, keine Verweigerungen, komplette Antwortdatensätze]) [17]. Deshalb wird hier statt des Begriffs der Repräsentativität der operationell zu definierende Begriff der Vergleichbarkeit verwendet. Vergleichbarkeit bezeichnet hier das Maß der Übereinstimmung zwei- er Studien hinsichtlich der Gesamtheit ihrer vergleichbar erhobenen Ergebnisse. Übereinstimmung kann dabei visuell/ thematisch beurteilt werden oder aber statistisch-objektiv, zum Beispiel als Anteil signifikanter Abweichungen in der Menge interessierender Charakteristika. Ist der Anteil der Abweichungen klein, so ist die Vergleichbarkeit gut. Die Vergleichbarkeit der verschiedenen LUCASWellen mit jeweils zeitnahen Hamburger Querschnittstudien wird in diesem Artikel in der oben beschriebenen Weise beurteilt. Dabei muss beachtet werden, dass einerseits jede LUCAS-Welle für sich genommen je eine Querschnittstudie darstellt, andererseits sukzessive Wellen jeweils dieselben Personen betreffen, was den Kohortencharakter der LUCAS-Studie ausmacht.

- Abb. 1 zeigt die für die Vergleiche herangezogenen Datenquellen. So wurden Daten der amtlichen Statistik zur älteren Hamburger Bevölkerung bzgl. Alter und Geschlecht mit denen aus vier LUCAS-Befragungswellen zu den Stichtagen 31.12.2000 (LUCAS Rekrutierung), 31.12.2007 (LUCAS-Welle 2), 31.12.2009 (LUCAS-Welle 3) und 31.12.2011 (LUCAS-Welle 4) verglichen. Darüber hinaus erfolgten qualitative Vergleiche $\mathrm{zu}$ drei repräsentativen Querschnittbefragungen, die vergleichbare Populationen aufgrund häufig verwendeter Merkmale beschreiben, die jeweils zeitnah zur jeweiligen LUCAS-Welle durchgeführt wurden: Befragung im Bezirk HamburgEimsbüttel im Jahr 2007 (LUCAS-Welle 2), Befragung in der Stadt Hamburg im Jahr 2009 (LUCAS-Welle 3) sowie Befragung im Bezirk Hamburg-Eimsbüttel im Jahr 2012 (LUCAS-Welle 4).

Die Rekrutierung der LUCAS-Teilnehmenden und sämtliche freiwillige Befragungswellen erfolgten gemäß der Deklaration von Helsinki nach Erteilung positiver Voten durch die Ethikkommission der Ärztekammer Hamburg und nach Abstimmung mit dem Hamburgischen Datenschutzbeauftragten. 
Tab. 1 Methodische Merkmale der vier Befragungen zur Gesundheit im Alter für vergleichende Analysen

\begin{tabular}{|c|c|c|c|c|}
\hline Merkmale & $\begin{array}{l}\text { LUCAS-Langzeit-Kohorte } \\
\text { Hamburg seit } 2000\end{array}$ & $\begin{array}{l}\text { Seniorenbefragung Bezirk } \\
\text { Hamburg-Eimsbüttel } 2007\end{array}$ & $\begin{array}{l}\text { Seniorenbefragung Bundes- } \\
\text { land Hamburg } 2009\end{array}$ & $\begin{array}{l}\text { Seniorenbefragung Bezirk } \\
\text { Hamburg-Eimsbüttel } 2012\end{array}$ \\
\hline Population & $\begin{array}{l}60 \text { Jahre und älter in LUCAS- } \\
\text { Hausarztpraxis }\end{array}$ & $\begin{array}{l}60 \text { Jahre und älter mit Wohn- } \\
\text { sitz Bezirk Hamburg-Eimsbüt- } \\
\text { tel }\end{array}$ & $\begin{array}{l}60 \text { Jahre und älter mit Wohn- } \\
\text { sitz Bundesland Hamburg }\end{array}$ & $\begin{array}{l}60 \text { Jahre und älter mit Wohn- } \\
\text { sitz Bezirk Hamburg-Eimsbüt- } \\
\text { tel }\end{array}$ \\
\hline \multirow[t]{2}{*}{$\begin{array}{l}\text { Erhebungs- } \\
\text { Methoden }\end{array}$} & $\begin{array}{l}\text { Wiederkehrende pseudony- } \\
\text { me schriftliche Befragung } \\
\text { mit Erinnerungsschreiben }\end{array}$ & $\begin{array}{l}\text { Anonyme schriftliche Befra- } \\
\text { gung des Gesundheitsamts } \\
\text { Eimsbüttel ohne Erinne- } \\
\text { rungsschreiben }\end{array}$ & $\begin{array}{l}\text { Telefoninterview durch Feld- } \\
\text { forschungsinstitut nach } \\
\text { schriftlicher Ankündigung } \\
\text { und ggf. Erinnerungsschreiben }\end{array}$ & $\begin{array}{l}\text { Anonyme schriftliche Befra- } \\
\text { gung des Gesundheitsamts } \\
\text { Eimsbüttel mit Erinnerungs- } \\
\text { schreiben }\end{array}$ \\
\hline & $\begin{array}{l}\text { Standardisierter Fragebo- } \\
\text { gen, je nach LUCAS-Welle } \\
\text { (W2-W4) 50-200 Variablen }\end{array}$ & $\begin{array}{l}\text { Standardisierter Fragebogen } \\
\text { mit } 47 \text { Variablen }\end{array}$ & $\begin{array}{l}\text { Standardisierte Befragung mit } \\
170 \text { Variablen }\end{array}$ & $\begin{array}{l}\text { Standardisierter Fragebogen } \\
\text { mit } 53 \text { Variablen }\end{array}$ \\
\hline \multirow{15}{*}{$\begin{array}{l}\text { Erhebungsinhalte } \\
\text { Variablen } \\
\text { (Charakteristika) }\end{array}$} & Soziodemografie & Soziodemografie & Soziodemografie & Soziodemografie \\
\hline & $\begin{array}{l}\text { Einstellungen zu Alter und } \\
\text { Gesundheit }\end{array}$ & $\begin{array}{l}\text { Einstellungen zu Alter und } \\
\text { Gesundheit }\end{array}$ & $\begin{array}{l}\text { Einstellungen zu Alter und } \\
\text { Gesundheit }\end{array}$ & $\begin{array}{l}\text { Einstellungen zu Alter und } \\
\text { Gesundheit }\end{array}$ \\
\hline & Selbstständigkeit & Selbstständigkeit & Selbstständigkeit & Selbstständigkeit \\
\hline & Mobilität & Mobilität & Mobilität & Mobilität \\
\hline & Stürze & Stürze & Stürze & Stürze \\
\hline & Ehrenamt & Ehrenamt & Ehrenamt & $\begin{array}{l}\text { Ehrenamt und Freizeitverhal- } \\
\text { ten }\end{array}$ \\
\hline & Rauchen & & Rauchen & Bewertung Wohnumfeld \\
\hline & Medikamente & & Medikamente & \\
\hline & $\begin{array}{l}\text { Gesundheitszustand, -ver- } \\
\text { halten, -vorsorge }\end{array}$ & & $\begin{array}{l}\text { Gesundheitszustand, -verhal- } \\
\text { ten, -vorsorge }\end{array}$ & \\
\hline & Sinne & & Sinne & \\
\hline & Schmerzen & & Schmerzen & \\
\hline & Stimmung & & Stimmung & \\
\hline & Soziales Netzwerk & & & \\
\hline & $\begin{array}{l}\text { Inanspruchnahme Gesund- } \\
\text { heitssystem }\end{array}$ & & & \\
\hline & $\begin{array}{l}\text { Funktioneller Status im } \\
\text { Verlauf }\end{array}$ & & & \\
\hline $\begin{array}{l}\text { Auswahlverfahren } \\
\text { der Stichproben }\end{array}$ & $\begin{array}{l}21 \text { Hausarztpraxen ge- } \\
\text { nerierten Listen mit al- } \\
\text { len Personen } 60+\text { Jahre } \\
\text { ( } n=9080) \text {. Gemäß Aus- } \\
\text { schlusskriterien (Pflegestu- } \\
\text { fe, Demenz, kein Deutsch) } \\
\text { verblieben } n=6986, \text { von } \\
\text { denen } n=3326 \text { schriftlich } \\
\text { zur Teilnahme einwilligten; } \\
9 \text { Mon. Rekrutierung ab } \\
10 / 2000\end{array}$ & $\begin{array}{l}\text { Zufallsauswahl durch das } \\
\text { HZM }^{1} \text { von } 2 \text { Stichproben à } \\
4 \% \text { (62.525 Senioren Bezirk } \\
\text { Eimsbüttel) aus } 14 \text { Teilstich- } \\
\text { proben mit } 7 \text { Altersklassen } \\
\text { (K1: } 60-64, \text { K7: } 90+\text { Jahre) } \\
\text { für Frauen und } 7 \text { für Männer: } \\
\text { zusammen } 2 \text { x } 2500 \text { Fälle; } \\
\text { davon angeschrieben: } n= \\
3342\end{array}$ & $\begin{array}{l}\text { Zufallsauswahl durch das } \\
\text { HZM }^{1} \text { von } 7833 \text { Personen in } \\
5 \text { Altersklassen (K1: 60-64, K2: } \\
65-69, \mathrm{~K} 3: 70-74, \mathrm{~K} 4: 75-79 \text {, } \\
\mathrm{K} 5: 80+) \text {, davon } n=5665 \mathrm{Zu}- \\
\text { fallsstichprobe inkl. Personen } \\
\text { mit Migrationshintergrund }{ }^{2} \\
\text { Erwarteter Rücklauf: jeweils } \\
200 \text { Personen pro Alters-Klasse } \\
1-5\end{array}$ & $\begin{array}{l}\text { Zufallsauswahl durch das } \\
\text { HZM }^{1} \text { von } 3 \text { Stichproben à } \\
100 \text { Fällen aus } 36 \text { Teilstich- } \\
\text { proben mit } 6 \text { Altersklassen } \\
\text { (K1: } 60-64, \text { K6: } 85+\text { Jahre) für } \\
\text { Frauen und } 6 \text { für Männer plus } \\
3 \text { Regionen (= } 36 \text { Teilstich- } \\
\text { proben): zusammen } 3 \times 3600 \\
\text { Fälle; davon angeschrieben: } \\
n=6858\end{array}$ \\
\hline
\end{tabular}

\section{Datenquellen}

\section{Datenquelle 1: Bevölkerungsdaten nach Alter und Geschlecht, Statistikamt Nord}

Für die Rekrutierung sowie für jede LUCAS-Welle erfolgten der Versand und der Rücklauf zu zwei Dritteln im ersten Jahr und einem Drittel im zweiten Jahr jeder Befragungswelle. Als Vergleichsjahr für den Abgleich mit den Bevölkerungsdaten des Statistikamtes wurde deshalb jeweils der 31. Dezember des ersten Jahres einer Welle festgelegt. Im Ergebnisteil dargestellt sind die Vergleiche zur LUCASRekrutierung [18] sowie zu den LUCASWellen 2 [19], 3 [20] und 4 [21]. Ein Vergleich mit LUCAS-Welle 1 erfolgte nicht, da sich diese direkt an die Rekrutierung anschloss (• Tab. 1).

Die verwendeten Statistikberichte inkl. der Bevölkerungsfortschreibungsdaten der Volkszählung im Jahr 1987 sind nach Alter und Geschlecht auf der Ho- mepage des Statistikamtes Nord (www. statistik-nord.de) verfügbar. Allerdings ist die Bevölkerung 90+ zusammengefasst, weshalb das Amt die Daten zu 90+ ergänzend in Fünfer-Jahrgängen (90-94, 95-99, 100+ Jahre) übermittelte, denn die Gliederung nach Einzeljahrgängen ist datenschutzrechtlich nicht gestattet. Ab 2011 wurde eine neue Datenbasis eingeführt (Zensus am 9.5.2011), die bundesweit eine geringere Bevölkerung ergibt [22]. Deshalb wurde für diesen 
Tab. 1 Methodische Merkmale der vier Befragungen zur Gesundheit im Alter für vergleichende Analysen (Fortsetzung)

\begin{tabular}{|c|c|c|c|c|}
\hline Merkmale & $\begin{array}{l}\text { LUCAS-Langzeit-Kohorte } \\
\text { Hamburg seit } 2000\end{array}$ & $\begin{array}{l}\text { Seniorenbefragung Bezirk } \\
\text { Hamburg-Eimsbüttel } 2007\end{array}$ & $\begin{array}{l}\text { Seniorenbefragung Bundes- } \\
\text { land Hamburg } 2009\end{array}$ & $\begin{array}{l}\text { Seniorenbefragung Bezirk } \\
\text { Hamburg-Eimsbüttel } 2012\end{array}$ \\
\hline \multirow[t]{5}{*}{$\begin{array}{l}\text { Ausschlüsse/ } \\
\text { Ausfälle und Aus- } \\
\text { schöpfungsquote }\end{array}$} & $\begin{array}{l}23 \% \text { Ausschluss gem. Kri- } \\
\text { terien (davon } 2 \% \text { ohne } \\
\text { ausreichende Deutsch- } \\
\text { kenntnisse) }\end{array}$ & $1 \%$ doppelte Adressen & $\begin{array}{l}6 \% \text { Ankündigung nicht zu- } \\
\text { stellbar }\end{array}$ & $1 \%$ Brief nicht zustellbar \\
\hline & $\begin{array}{l}\text { Ausschöpfung: } 48 \% \text { ( } n= \\
\text { 3326) }\end{array}$ & $2 \%$ Brief nicht zustellbar & $\begin{array}{l}48 \% \text { Ausschluss: kein Telefon- } \\
\text { bucheintrag }\end{array}$ & \\
\hline & Welle 2 Ausschöpfung: $77 \%$ & $\begin{array}{l}\text { Ausschöpfung: } 29 \% \text { ( } n= \\
950)\end{array}$ & & \\
\hline & Welle 3 Ausschöpfung: $88 \%$ & & $\begin{array}{l}\text { Ausschöpfung von } n=5665 \text { : } \\
19 \% \text { bzw. } 37 \% \text { Teilnahme } \\
\text { aller Personen mit Telefonan- } \\
\text { schluss ( } n=1098 \text { gewichtete } \\
\text { Angaben) }\end{array}$ & \\
\hline & \multicolumn{2}{|l|}{ Welle 4 Ausschöpfung: 90 \% } & & $\begin{array}{l}\text { Ausschöpfung } 29 \% \text { ( } n= \\
2000 \text { ) bzw. } n=1936 \text { für ge- } \\
\text { wichtete Angaben }\end{array}$ \\
\hline $\begin{array}{l}\text { Datenreduktion } \\
\text { für vergleichende } \\
\text { Analysen }\end{array}$ & $\begin{array}{l}\text { Welle 2: Alle 67+ Jahre mit } \\
\text { Wohnort Eimsbüttel }(n= \\
756) \\
\text { Welle 3: Alle 70+ Jahre mit } \\
\text { Wohnort Hamburg }(n= \\
\text { 1542) } \\
\text { Welle 4: Alle 71+ Jahre mit } \\
\text { Wohnort Eimsbüttel ( } n= \\
\text { 544) }\end{array}$ & $\begin{array}{l}\text { Bezirk Hamburg-Eimsbüttel: } \\
\text { Datensatz mit Mindestalter } \\
67 \text { Jahre }(n=611)\end{array}$ & $\begin{array}{l}\text { Stadt Hamburg: Gewichteter } \\
\text { Datensatz der Zufallsstichpro- } \\
\text { be mit Mindestalter } 70 \text { Jahre }^{3} \\
(n=574)\end{array}$ & $\begin{array}{l}\text { Bezirk Hamburg-Eimsbüttel: } \\
\text { Gewichteter Datensatz mit } \\
\text { Mindestalter } 71 \text { Jahre }(n= \\
\text { 999) }\end{array}$ \\
\hline Referenz & Dapp et al. 2012 [31] & Dapp et al. 2009 [32] & FHH 2011 [33] & Dapp et al. 2013 [34] \\
\hline \multicolumn{5}{|c|}{$\begin{array}{l}\text { 'HZM Hamburger Fachamt für Zentrale Meldeangelegenheiten } \\
{ }^{2} \text { Die Befragung der Stadt Hamburg enthält zusätzlich eine nicht-repräsentative Migrantenstichprobe ( } n=2168 \text { ) ohne Berücksichtigung in diesem Artikel, } \\
\text { da nicht repräsentativ } \\
{ }^{3} \text { Entscheidung für Vergleiche ab Mindestalter } 70 \text { Jahre (nicht } 69 \text { Jahre), da in Befragung Stadt Hamburg größter Gewichtungsfaktor für Altersgruppe } \\
65-69 \text { Jahre, vgl. [33]; S. 20: kleiner/gleich } 64 \text { Jahre: 1,12; 65-69 Jahre: 1,27; 70-74 Jahre: 0,94; 75-79 Jahre: 0,68; } 80 \text { + Jahre: } 1,01\end{array}$} \\
\hline
\end{tabular}

Artikel die Datenbasis im Jahr 2011 nicht gewechselt [21].

\section{Datenquelle 2: LUCAS- Langzeitkohortenstudie (Albertinen-Haus Hamburg)}

Basis der LUCAS-Langzeitkohorte bildet die multizentrische europäische Studie PRO-AGE, für die im Jahr 2000 selbstständig lebende Menschen ab 60 Jahren über Hamburger Hausarztpraxen rekrutiert wurden. Es willigten 21 Hausarztpraxen ein und lieferten Listen aller ihrer mindestens 60-jährigen Patienten. Danach wurden alle ausgeschlossen mit einer Pflegestufe gem. MDK, einer Abhängigkeit in Aktivitäten des täglichen Lebens (ADL), kognitiven Auffälligkeiten (Mini Mental Status Test $\leq 24$ [23]), terminaler Erkrankung und unzureichenden Deutschkenntnissen. Alle Verbliebenen $(n=6986)$ erhielten aus ihrer Hausarztpraxis ein Informationsschrei- ben zur Teilnahme, ein Einwilligungsformular sowie einen Kurzfragebogen. Die Rekrutierungszeit umfasste neun Monate. Der Vergleich zwischen den teilnehmenden und ablehnenden Personen einer der 21 Hausarztpraxen ergab keine signifikanten Unterschiede bezüglich Alter, Geschlecht und Komorbidität (metabolisches Syndrom) [24].

Während der PRO-AGE-Studie wurde eine Intervention in Kleingruppen durch ein Gesundheitsberaterteam am geriatrischen Zentrum [25] oder als präventiver Hausbesuch durch eine Fachpflegekraft [26] in randomisiertkontrolliertem Studiendesign angeboten [15, 27]. Bis Ende 2011 bestand für die Hausärzte ein akkreditierter, curricularer hausärztlicher Qualitätszirkel mit präventivmedizinischen Inhalten, sodass die Hausärzte über aktuelle Kenntnisse zu Alterung, Altersmedizin, Assessment und Prävention verfügen [28].
Die Baseline-Merkmale wurden per Kurzfragebogen [29], ergänzt um die Angabe der Pflegestufe, erhoben, gefolgt von einem standardisierten Fragebogen (Health Risk Appraisal) zu Welle 1 [28]. Die Variablenerhebung fokussierte nicht auf singuläre Erkrankungen (Diagnosen), sondern erfasste besonders gesundheitsrelevante Dimensionen zum Altern, die bekannte Risikofaktoren für die Verschlechterung des funktionellen Status selbstständig lebender älterer Menschen darstellen, wie z. B. geringe körperliche Aktivität, Fehlernährung, Rauchen, soziale Isolation oder Sehminderung [30]. In den folgenden LUCASWellen wurden Fragebögen eingesetzt, die weiterhin alle ursprünglichen Themenbereiche umfassten, jedoch um Forschungsschwerpunkte erweitert wurden. Ein Satz an Kernfragen blieb im Verlauf stets unverändert (• Tab. 1). Die Variablen jeder LUCAS-Welle, deren 
Zuordnung $\mathrm{zu}$ gesundheitsrelevanten Dimensionen sowie die quantitativ hohe Beteiligung im Langzeitverlauf (Ausfallprozess) wurden im Detail beschrieben [31]. Die Datenbasis der LUCAS-Kohorte beinhaltet Informationen von 3326 Personen über maximal 12 Jahre (entspricht 30.000 Personenjahren). Die Informationen zur jeweiligen Teilnahmerate der einzelnen LUCAS-Wellen enthält • Abb. 1.

\section{Datenquelle 3: Seniorenbefragung 2007 (Gesundheitsamt Bezirk Hamburg-Eimsbüttel)}

Zur Ermittlung der Infrastrukturbedarfe der älteren Bevölkerung und der Inanspruchnahme von Gesundheitsangeboten wurde diese Erhebung für die Kommunalplanung Eimsbüttel (ca. 250.000 Einwohner) initiiert. Für die repräsentative Stichprobenziehung wurde die 60jährige und ältere Bezirksbevölkerung in 14 Teilstichproben mit sieben 5-JahresAltersklassen für Frauen und für Männer untergliedert. Durch das Hamburger Fachamt für Zentrale Meldeangelegenheiten (HZM) wurden in allen 14 Teilstichproben zwei $4 \%$-Zufallsstichproben à 2500 Fälle gezogen. Der unterdurchschnittliche Rücklauf in den Teilstichproben ab 80 Jahre (1. Ziehung) wurde mit der Auswahl gleich alter und gleichgeschlechtlicher Personen der 2. Ziehung ausgeglichen. Die Datenerhebung erfolgte von März bis Mai 2007. Die Methodik und Ergebnisse sind im Detail beschrieben [32]. Neben soziodemografischen Angaben wurden mittels standardisiertem Selbstausfüllerbogen Einstellungen zum Altern erfragt sowie Angaben dazu, was ältere Bürger selbst für Gesundheit, Mobilität und Selbstständigkeit tun (•Tab. 1). Angaben zum Rücklauf zeigt • Abb. 1.

\section{Datenquelle 4: Seniorenbefragung 2009 (Behörde für Gesundheit und Verbraucherschutz Hamburg)}

Das Ziel der Befragung „Gesundheit älterer Menschen in Hamburg " waren die Erhebung, Beschreibung und Bewertung von Informationen zur Gesundheit bei einer altersrepräsentativen Stichprobe, um auf dieser Grundlage gesundheitsfördernde und präventive
Maßnahmen für die ältere Hamburger Bevölkerung zielgerecht zu verbessern. Diese Befragung (Mai bis September 2009) war ein Teilprojekt des LUCASForschungsverbundes [12]. Per Anschreiben wurde eine Zufallsauswahl des HZM der Hamburger Bevölkerung ab 60 Jahren mit Telefonbucheintrag (50\% der älteren Bevölkerung in Hamburg) über die Befragung informiert. Danach erfolgte ein Anruf durch das beauftragte Feldforschungsinstitut, das um Teilnahme bat und die Datenerhebung durchführte (•Abb. 1). Die Befragung wurde in Anlehnung an die Telefonumfragen des Robert Koch-Instituts sowie den schriftlichen Fragebogen der LUCAS-Langzeitkohorte entwickelt und auf Deutsch, Englisch, Französisch, Türkisch und Polnisch im Telefoninterview angeboten. Diese Daten wurden für fünf Altersklassen gewichtet. Gewichtungsfaktoren, Methodik und Ergebnisse sind im Detail beschrieben [33]. Die standardisierte Erfassung enthielt über 100 Einzelfragen zu verschiedenen Gesundheitsdimensionen (•Tab. 1).

\section{Datenquelle 5: Seniorenbefragung 2012 (Gesundheitsamt Bezirk Hamburg-Eimsbüttel)}

Die zweite anonyme Querschnittbefragung wurde von Juli bis September 2012 erneut vom Gesundheitsamt Eimsbüttel durchgeführt. Da die Befragung im Jahr 2007 regionale Unterschiede gezeigt hatte und die Nutzung der Infrastruktur des Wohnumfelds interessierte, wurde die Anzahl der Teilstichproben durch folgende Modifikationen auf 36 erhöht: Neuaufnahme des Unterscheidungsmerkmals „3 Regionen im Bezirk“ bei Reduktion der Gruppen „Alter“ von sieben auf sechs durch Bildung der höchsten Altersgruppe „85+ Jahre“. Das HZM zog in allen Teilstichproben zufällig je 100 Personen (3600 Fälle) (• Tab. 1). Für repräsentative Ergebnisse wurde der Datensatz nach den Variablen Region (3), Altersklassen (6) und Geschlecht (2) gewichtet [34].

Die Mehrzahl der Fragen von 2007 wurden 2012 erneut gestellt und ergänzt um mobilitätsrelevante Themen (Erleben von Stadtteil und Wohnumfeld, Durch- führung von Freizeitaktivitäten im Aktionsraum) (• Tab. 1).

\section{Vorbereitung vergleichender Analysen durch Reduktion des Datenumfangs}

Mit den zwei Eimsbütteler Befragungen (2007, 2012) wurden die Ergebnisse der im Bezirk Eimsbüttel wohnhaften LUCAS-Teilnehmenden verglichen.

Der Vergleich der Hamburger Befragung im Jahr 2009 mit der LUCAS-Kohorte erfolgte lediglich ab dem Alter von 70 Jahren, obschon die LUCAS-Kohorte $\mathrm{zu}$ diesem Zeitpunkt ein Mindestalter von 69 Jahren aufwies. Dies machte Korrekturen aufgrund von Gewichtungen überflüssig.

- Tab. 1 fasst die Angaben zu den Populationen der vier Seniorenbefragungen, Erhebungsmethoden und -inhalte zusammen, inkl. Auswahlverfahren der Stichproben, Ausschlüsse, Ausfälle sowie zur Datensatzreduktion für die vergleichende Ergebnisdarstellung.

\section{Datenanalyse und Ergebnis- darstellung}

Zum Vergleich der Altersverteilungen nach Geschlecht zwischen den LUCASWellen 1-4 und den Daten des Statistikamtes Nord wurde die in der Demografie übliche Pyramidendarstellung verwendet. Dabei wurde in der linken Hälfte jeder Pyramide die Zahl der Männer pro Altersgruppe in LUCAS (blau) sowie in derselben Pyramidenhälfte die Altersgruppen der Hamburger Männer (weiß) dargestellt. Für den Vergleich wurden die Zahlen des Statistischen Amtes mittels geschlechtsspezifischer Faktoren auf die Größe der LUCAS-Stichprobe heruntergerechnet. Die so errechneten Anzahlen entsprechen in jeder Altersgruppe der Anzahl Männer, die in der LUCASStudie gemäß der Hamburger Statistik zu erwarten waren. Analog wurden die Zahlen für Frauen (rot) jeweils auf der rechten Pyramidenhälfte behandelt (• Abb. 2a-d).

Unterschiede bezüglich soziodemografischer und gesundheitlicher Kennzahlen wurden auf ihre Signifikanz geprüft. Alter, Medikamentenzahl und 
Tab. 2 Inhaltliche Merkmale (Auswahl) identischer Fragestellungen in drei Befragungswellen der LUCAS-Langzeit-Kohortenstudie und drei Hamburger Querschnitt-Befragungen (LUCAS-Welle 2 vs. Befragung Eimsbüttel 2007, LUCAS-Welle 3 vs. Befragung Hamburg 2009, LUCAS-Welle 4 vs. Befragung Eimsbüttel 2012)

\begin{tabular}{|c|c|c|c|c|c|c|c|c|c|}
\hline $\begin{array}{l}\text { Merkmale (Auswahl) } \\
\text { Identische Fragen in } \\
\text { repetitiver LUCAS-Lang- } \\
\text { zeitkohortenbefragung } \\
\text { und drei Hamburger } \\
\text { Querschnittbefragun- } \\
\text { gen mit Anspruch auf } \\
\text { Repräsentativität }\end{array}$ & $\begin{array}{l}\text { LUCAS-Kohorte } \\
\text { nur Eimsbüttel } \\
2007 / 08 \text { (W2) } \\
\text { Mindestalter: } \\
67+\text { Jahre } \\
n=756\end{array}$ & $\begin{array}{l}\text { Repräsen- } \\
\text { tative } \\
\text { Befragung } \\
\text { Eimsbüttel } \\
2007 \text { Alle } \\
\text { Befragten } \\
67+\text { Jahre } \\
n=611\end{array}$ & $\begin{array}{l}p- \\
\text { Wert }^{1}\end{array}$ & $\begin{array}{l}\text { LUCAS-Kohorte } \\
\text { gesamt Ham- } \\
\text { burg } \\
2009 / 10 \text { (W3) } \\
\text { Mindestalter: } \\
\text { 70+ Jahre } \\
n=1542\end{array}$ & $\begin{array}{l}\text { Repräsen- } \\
\text { tative } \\
\text { Befragung } \\
\text { Hamburg } \\
2009 \text { Alle } \\
\text { Befragten } \\
\text { 70+ Jahre } \\
n=574\end{array}$ & $\begin{array}{l}\text { p- } \\
\text { Wert }\end{array}$ & $\begin{array}{l}\text { LUCAS-Kohorte } \\
\text { nur Eimsbüttel } \\
2011 / 12 \text { (W4) } \\
\text { Mindestalter: } \\
\text { 71+ Jahre } \\
n=544\end{array}$ & $\begin{array}{l}\text { Repräsen- } \\
\text { tative } \\
\text { Befragung } \\
\text { Eimsbüttel } \\
2012 \text { Alle } \\
\text { Befragten } \\
71+\text { Jahre } \\
n=999\end{array}$ & $\begin{array}{l}p- \\
\text { Wert }^{1}\end{array}$ \\
\hline \multicolumn{10}{|l|}{$\begin{array}{l}\text { Soziodemografische } \\
\text { Merkmale }\end{array}$} \\
\hline $\begin{array}{l}\text { Alter zur Befragung in } \\
\text { Jahren: Mittelwert (MW) } \\
\text { (Minimum-Maximum) }\end{array}$ & $76,3(67,1-99,7)$ & $\begin{array}{l}75,6 \\
(67,0-97,0)\end{array}$ & 0,048 & $\begin{array}{l}77,9 \\
(70,0-100,7)\end{array}$ & $\begin{array}{l}77,1 \\
(70-90)\end{array}$ & $--^{3}$ & $\begin{array}{l}79,0 \\
(71,3-100,9)\end{array}$ & $\begin{array}{l}78,6 \\
(71,0-100,8)\end{array}$ & $-{ }^{3}$ \\
\hline Frauen: $n(\%)$ & $461 / 756(61,0)$ & $\begin{array}{l}363 / 607 \\
(59,8)\end{array}$ & 0,659 & $968 / 1542(62,8)$ & $\begin{array}{l}359 / 574 \\
(62,5)\end{array}$ & $-{ }^{3}$ & $332 / 544(61,0)$ & $\begin{array}{l}604 / 999 \\
(60,5)\end{array}$ & $-{ }^{3}$ \\
\hline $\begin{array}{l}\text { Geburtsort in Deutschland: } \\
n(\%)\end{array}$ & $728 / 753(96,7)$ & $\begin{array}{l}587 / 607 \\
(96,7)\end{array}$ & 0,979 & $\begin{array}{l}1492 / 1541 \\
(96,8)\end{array}$ & $\begin{array}{l}554 / 574 \\
(96,6)\end{array}$ & 0,690 & $528 / 544(97,1)$ & $\begin{array}{l}957 / 993 \\
(96,3)\end{array}$ & 0,215 \\
\hline Allein lebend: $n$ (\%) & $281 / 748(37,6)$ & $\begin{array}{l}233 / 603 \\
(38,6)\end{array}$ & 0,686 & $544 / 1429(38,1)$ & $\begin{array}{l}250 / 574 \\
(43,5)\end{array}$ & 0,058 & $221 / 536(41,2)$ & $\begin{array}{l}427 / 994 \\
(42,9)\end{array}$ & 0,204 \\
\hline $\begin{array}{l}\text { Caregiver vorhanden im } \\
\text { Bedarfsfall: } n(\%)\end{array}$ & $620 / 744(83,3)$ & $\begin{array}{l}527 / 606 \\
(87,0)\end{array}$ & 0,063 & $\begin{array}{l}1230 / 1455 \\
(84,5)\end{array}$ & $\begin{array}{l}479 / 574 \\
(83,4)\end{array}$ & 0,550 & $467 / 539(86,6)$ & $\begin{array}{l}847 / 979 \\
(86,5)\end{array}$ & 0,384 \\
\hline $\begin{array}{l}\text { Höherer Schulabschluss } \\
\text { Abitur/Realschule: } n(\%)\end{array}$ & $282 / 709(39,8)$ & $\begin{array}{l}266 / 605 \\
(44,0)\end{array}$ & 0,124 & $591 / 1445(40,9)$ & $\begin{array}{l}284 / 574 \\
(49,6)\end{array}$ & 0,000 & $210 / 515(40,8)$ & $\begin{array}{l}466 / 986 \\
(47,3)\end{array}$ & 0,012 \\
\hline $\begin{array}{l}\text { Abgeschlossene akademi- } \\
\text { sche Ausbildung: } n(\%)\end{array}$ & $86 / 684(12,6)$ & $\begin{array}{l}76 / 592 \\
(12,8)\end{array}$ & 0,887 & - & - & & $64 / 500(12,8)$ & $\begin{array}{l}123 / 975 \\
(12,6)\end{array}$ & 0,894 \\
\hline \multicolumn{10}{|l|}{ Mobilität im Aktionsraum } \\
\hline $\begin{array}{l}\text { Autofahrer Selbstfahrer/ } \\
\text { Mitfahrer: } n(\%)\end{array}$ & $484 / 745(65,0)$ & $\begin{array}{l}350 / 608 \\
(57,6)\end{array}$ & 0,005 & $950 / 1452(65,4)$ & $\begin{array}{l}386 / 574 \\
(67,2)\end{array}$ & 0,394 & $341 / 523(65,2)$ & $\begin{array}{l}590 / 991 \\
(59,5)\end{array}$ & 0,000 \\
\hline $\begin{array}{l}\text { Nutzung öffentl. Nahver- } \\
\text { kehr mind. an } 5 \text { Tagen in } \\
\text { der Woche: } n(\%)\end{array}$ & $135 / 744(18,1)$ & $\begin{array}{l}126 / 532 \\
(23,7)\end{array}$ & 0,001 & $327 / 1449(22,6)$ & $\begin{array}{l}109 / 574 \\
(19,1)\end{array}$ & 0,119 & - & - & \\
\hline $\begin{array}{l}\text { Fahrradfahrer regelmäßig/ } \\
\text { gelegentlich: } n(\%)\end{array}$ & $354 / 749(47,3)$ & $\begin{array}{l}287 / 601 \\
(47,8)\end{array}$ & 0,858 & $649 / 1533(42,3)$ & $\begin{array}{l}251 / 574 \\
(43,6)\end{array}$ & 0,331 & $214 / 543(39,4)$ & $\begin{array}{l}457 / 990 \\
(46,2)\end{array}$ & 0,587 \\
\hline $\begin{array}{l}\text { Keine Sturzangst: } n \\
(\%)\end{array}$ & $517 / 741(69,8)$ & $\begin{array}{l}385 / 600 \\
(64,2)\end{array}$ & 0,030 & $999 / 1526(65,5)$ & $\begin{array}{l}401 / 574 \\
(69,8)\end{array}$ & 0,030 & $372 / 543(68,5)$ & $\begin{array}{l}644 / 979 \\
(65,7)\end{array}$ & 0,000 \\
\hline $\begin{array}{l}\text { Letzte } 12 \text { Monate hingefal- } \\
\text { len: } n(\%)\end{array}$ & $209 / 744(28,1)$ & $\begin{array}{l}214 / 606 \\
(35,3)\end{array}$ & 0,004 & $457 / 1538(29,7)$ & $\begin{array}{l}139 / 574 \\
(24,1)\end{array}$ & 0,004 & $151 / 542(27,9)$ & $\begin{array}{l}341 / 987 \\
(34,5)\end{array}$ & 0,000 \\
\hline Ehrenamt: $n(\%)$ & $82 / 736(11,1)$ & $\begin{array}{l}86 / 593 \\
(14,5)\end{array}$ & 0,067 & $174 / 1508(11,5)$ & $\begin{array}{l}88 / 574 \\
(15,4)\end{array}$ & 0,021 & $63 / 544(11,6)$ & $\begin{array}{l}137 / 946 \\
(14,4)\end{array}$ & 0,812 \\
\hline \multicolumn{10}{|l|}{$\begin{array}{l}\text { Gesundheit und Selbst- } \\
\text { ständigkeit }\end{array}$} \\
\hline $\begin{array}{l}\text { Selbstempfundener Allge- } \\
\text { meinzustand sehr gut/gut: } \\
n(\%)\end{array}$ & $467 / 742(62,9)$ & $\begin{array}{l}379 / 603 \\
(62,9)\end{array}$ & 0,974 & $987 / 1524(64,7)$ & $\begin{array}{l}336 / 574 \\
(58,6)^{4}\end{array}$ & 0,007 & $342 / 539(63,5)$ & $\begin{array}{l}613 / 990 \\
(61,9)\end{array}$ & 0,018 \\
\hline $\begin{array}{l}\text { Kein Krankenhausauf- } \\
\text { enthalt in den letzten } \\
12 \text { Monaten: } n(\%)\end{array}$ & $539 / 752(71,7)$ & $\begin{array}{l}448 / 601 \\
(74,5)\end{array}$ & 0,238 & $\begin{array}{l}1063 / 1450 \\
(73,3)\end{array}$ & $\begin{array}{l}423 / 574 \\
(73,7)\end{array}$ & 0,732 & $366 / 540(67,8)$ & $\begin{array}{l}697 / 990 \\
(70,3)\end{array}$ & 0,955 \\
\hline Pflegestufe (1-3): n (\%) & $37 / 746(5,0)^{5}$ & $42 / 589(7,1)$ & 0,095 & $93 / 1521(6,1)$ & $28 / 574(4,9)$ & 0,202 & $38 / 538(7,1)$ & $72 / 973(7,4)$ & 0,058 \\
\hline $\begin{array}{l}\text { Baden, selbstständig ohne } \\
\text { Schwierigkeiten: } n(\%)\end{array}$ & & & & $\begin{array}{l}1225 / 1464 \\
(83,7)\end{array}$ & $\begin{array}{l}503 / 574 \\
(87,7)\end{array}$ & 0,014 & & & \\
\hline $\begin{array}{l}500 \text { m gehen selbstständig } \\
\text { ohne Schwierigkeiten: } n \\
\text { (\%) }\end{array}$ & & & & $\begin{array}{l}1081 / 1457 \\
(74,2)\end{array}$ & $\begin{array}{l}488 / 574 \\
(85,1)\end{array}$ & 0,000 & & & \\
\hline $\begin{array}{l}\text { Schmerzen, die nie ganz } \\
\text { verschwinden: } n(\%)\end{array}$ & & & & $590 / 1422(41,5)$ & $\begin{array}{l}239 / 574 \\
(41,7)\end{array}$ & 0,908 & & & \\
\hline
\end{tabular}


Tab. 2 Inhaltliche Merkmale (Auswahl) identischer Fragestellungen in drei Befragungswellen der LUCAS-Langzeit-Kohortenstudie und drei Hamburger Querschnitt-Befragungen (LUCAS-Welle 2 vs. Befragung Eimsbüttel 2007, LUCAS-Welle 3 vs. Befragung Hamburg 2009, LUCAS-Welle 4 vs. Befragung Eimsbüttel 2012) (Fortsetzung)

\begin{tabular}{|c|c|c|c|c|c|c|c|c|c|}
\hline $\begin{array}{l}\text { Merkmale (Auswahl) } \\
\text { Identische Fragen in } \\
\text { repetitiver LUCAS-Lang- } \\
\text { zeitkohortenbefragung } \\
\text { und drei Hamburger } \\
\text { Querschnittbefragun- } \\
\text { gen mit Anspruch auf } \\
\text { Repräsentativität }\end{array}$ & $\begin{array}{l}\text { LUCAS-Kohorte } \\
\text { nur Eimsbüttel } \\
2007 / 08 \text { (W2) } \\
\text { Mindestalter: } \\
67+\text { Jahre } \\
n=756\end{array}$ & $\begin{array}{l}\text { Repräsen- } \\
\text { tative } \\
\text { Befragung } \\
\text { Eimsbüttel } \\
2007 \text { Alle } \\
\text { Befragten } \\
67+\text { Jahre } \\
n=611\end{array}$ & $\begin{array}{l}\text { p- } \\
\text { Wert }\end{array}$ & $\begin{array}{l}\text { LUCAS-Kohorte } \\
\text { gesamt Ham- } \\
\text { burg } \\
2009 / 10 \text { (W3) } \\
\text { Mindestalter: } \\
70+\text { Jahre } \\
n=1542\end{array}$ & $\begin{array}{l}\text { Repräsen- } \\
\text { tative } \\
\text { Befragung } \\
\text { Hamburg } \\
2009 \text { Alle } \\
\text { Befragten } \\
\text { 70+ Jahre } \\
n=574\end{array}$ & $\begin{array}{l}p \text { - } \\
\text { Wert }\end{array}$ & $\begin{array}{l}\text { LUCAS-Kohorte } \\
\text { nur Eimsbüttel } \\
\text { 2011/12 (W4) } \\
\text { Mindestalter: } \\
\text { 71+ Jahre } \\
n=544\end{array}$ & $\begin{array}{l}\text { Repräsen- } \\
\text { tative } \\
\text { Befragung } \\
\text { Eimsbüttel } \\
2012 \text { Alle } \\
\text { Befragten } \\
71+\text { Jahre } \\
n=999\end{array}$ & $\begin{array}{l}p \text { - } \\
\text { Wert }^{1}\end{array}$ \\
\hline $\begin{array}{l}\text { Anzahl verschreibungs- } \\
\text { pflichtige Medikamente: } \\
\text { MW (Min-Max) }\end{array}$ & & & & $3,7(0-17)$ & $3,5(0-20)$ & 0,069 & & & \\
\hline $\begin{array}{l}\text { BMI: MW (Mini- } \\
\text { mum-Maximum) }\end{array}$ & & & & $26,2(14-51)$ & $\begin{array}{l}26,0 \\
(16-51)\end{array}$ & 0,572 & & & \\
\hline Zigarettenkonsum: $n$ (\%) & & & & $101 / 1380(7,3)$ & $35 / 574(6,1)$ & 0,314 & & & \\
\hline $\begin{array}{l}\text { Hilfsmittel zur Fortbewe- } \\
\text { gung nutzen: } n(\%)\end{array}$ & & & & $309 / 1455(21,2)$ & $\begin{array}{l}116 / 574 \\
(20,2)\end{array}$ & 0,419 & & & \\
\hline $\begin{array}{l}\text { Leichte Einkäufe, selbst- } \\
\text { ständig ohne Schwierigkei- } \\
\text { ten: } n(\%)\end{array}$ & & & & $\begin{array}{l}1153 / 1455 \\
(79,2)\end{array}$ & $\begin{array}{l}495 / 574 \\
(86,3)\end{array}$ & 0,000 & & & \\
\hline $\begin{array}{l}\text { Sehvermögen, ausgezeich- } \\
\text { net/gut: } n(\%)\end{array}$ & & & & $944 / 1445(65,3)$ & $\begin{array}{l}402 / 574 \\
(69,9)\end{array}$ & 0,023 & & & \\
\hline $\begin{array}{l}\text { Hörvermögen, ausgezeich- } \\
\text { net/gut: } n(\%)\end{array}$ & & & & $872 / 1438(60,7)$ & $\begin{array}{l}382 / 574 \\
(66,7)\end{array}$ & 0,014 & & & \\
\hline \multicolumn{10}{|l|}{ Gesundheitsvorsorge } \\
\hline $\begin{array}{l}\text { Zahnkontrolle im letzten } \\
\text { Jahr: } n(\%)\end{array}$ & & & & $\begin{array}{l}1253 / 1438 \\
(87,1)\end{array}$ & $\begin{array}{l}436 / 574 \\
(75,9)\end{array}$ & 0,000 & & & \\
\hline $\begin{array}{l}\text { Regelmäßige Teilnahme } \\
\text { an Grippeschutzimpfung: } n \\
(\%)\end{array}$ & $552 / 735(75,1)$ & $\begin{array}{l}360 / 567 \\
(63,5)\end{array}$ & 0,000 & $\begin{array}{l}1014 / 1246 \\
(81,4)\end{array}$ & $\begin{array}{l}347 / 574 \\
(60,4)\end{array}$ & 0,000 & - & $\begin{array}{l}582 / 920 \\
(63,3)\end{array}$ & \\
\hline \multicolumn{10}{|c|}{$\begin{array}{l}{ }^{1} \text { 2-seitige p-Werte; für Anzahlen basierend auf dem Chiquadrat-Test, bei gewichteten Studien nach McFadden et al. [35]; für Mittelwertsvergleiche basierend } \\
\text { auf dem t-Test } \\
{ }^{2} \text { Entscheidung für Mindestalter } 70 \text { Jahre (nicht } 69 \text { Jahre), vgl. Fußnote } 3 \text { in • Tab. } 1 \\
{ }^{3} \text { Unterschiede in Alter und Geschlecht wurden hier nicht getestet, da sich bei gewichteten Studien jene Faktoren, die die Gewichtung bestimmten, nicht } \\
\text { konsistent testen lassen [35] } \\
{ }^{4} \text { Antwortmöglichkeiten Befragung Hamburg 2009: sehr gut, gut, weder gut noch schlecht, schlecht, sehr schlecht (5er Skala) im Unterschied zu Antwort- } \\
\text { möglichkeiten in allen anderen Befragungen LUCAS und Eimsbüttel: sehr gut, gut, mäßig, schlecht (4er Skala) } \\
{ }^{5} \text { Zur LUCAS-Rekrutierung (7 Jahre früher) war Pflegebedürftigkeit Ausschlusskriterium }\end{array}$} \\
\hline
\end{tabular}

Body-Mass-Index (BMI) wurden mit dem $\mathrm{t}$-Test verglichen und Anteile mittels Chiquadrat-Test. Beiden gewichteten Querschnittstudien (Hamburg 2009 und Hamburg 2012) wurden statistische Unterschiede in Alter und Geschlecht nicht getestet, da sich Anteile von Faktoren, die die Gewichtung bestimmten, nicht konsistent vergleichen lassen [35]. Diese geschichteten Zufallsstichproben (keine Quotenstichproben [17]), bei denen z. B. in jeder Altersgruppe dieselbe Anzahl von Personen angeschrieben wurde, beeinflussen die rohen Anzahlen und damit die Anteile in den Kategorien derjenigen Faktoren, die die Schichtung bestimmten. Um das zu korrigieren, wurden gewichtete Anzahlen errechnet. Jedoch repräsentieren die gewichteten Anzahlen und die daraus berechneten Anteile die Verhältnisse in der Population, nach der gewichtet wurde, und nicht diejenigen in der Stichprobe.

Verglichen wurde jeweils die zeitlich am besten passende LUCAS-Welle mit einer der Querschnittstudien. Da mögliche Unterschiede von Interesse waren, wurde auf eine Bonferroni-Holms-Korrektur der $p$-Werte verzichtet (•Tab. 2).

\section{Ergebnisse}

Die Abb. 2a-d zeigen vier vergleichende Bevölkerungspyramiden (in 5-Jahres-
Altersklassen getrennt nach Geschlecht) der älteren Hamburger Bevölkerung und der LUCAS-Kohorte zu LUCAS-Rekrutierung/Welle 1 (2000) sowie zu LUCASWellen 2 (2007), 3 (2009) und 4 (2011).

Der Vergleich zeigt eine gute Übereinstimmung für alle vier Wellen (- Abb. 2a-d). Aufgrund des LUCASRekrutierungsbeginns im Oktober 2000 gilt nur eine bedingte zeitliche Vergleichbarkeit. Die Pyramide zur LUCASRekrutierung (2000) zeigt leicht überproportionale Anteile beider Geschlechter in den Altersgruppen ab 65 bis unter 85 Jahre, für die Männer inkl. 85 bis unter 90 Jahre. Untervertreten sind Männer der Altersgruppe 60 bis 64 , vermutlich weil 
diese, noch im Arbeitsprozess, seltener eine Hausarztpraxis aufsuchten. Bei den Frauen sind die Hochalten ab 85 Jahren untervertreten, was wohl auf die LUCAS-Einschlussbedingung „selbständig, nicht pflegebedürftig“ zurückzuführen ist. Diese Diskrepanzen verschwinden weitgehend in den Wellen 2 bis 4, denn der Aufbau nach Alter und Geschlecht der LUCAS-Kohorte näherte sich im Zeitverlauf demjenigen der allgemeinen Bevölkerung. Mit der Alterung der Kohorte wuchs der Anteil Hochalter verglichen mit den amtlichen Angaben zur Hamburger Bevölkerung in diesen Altersklassen (• Abb. 2a-d).

Es sind Diskrepanzen zwischen den Daten der amtlichen Melderegister und dem Statistikamt zu berücksichtigen. Die vom Statistikamt Nord bestätigte Differenz der mindestens 60-Jährigen der Hamburger Bevölkerung zwischen den Hochrechnungsdaten der Volkszählung 1987 zum Stichtag 31.12.2011 (430.827) [21] und dem Zensus 2011 (416.249) [22] in Höhe von 14.398 Personen relativiert die Verluste durch Todesfälle und unbekannt Verzogene im LUCAS-Langzeitverlauf.

Für die hohe Verlässlichkeit der LUCAS-Angaben spricht, dass die Teilnehmenden von ihren gut bekannten Hausarztpraxen rekrutiert wurden. Außerdem wurden die Studienteilnehmenden jährlich durch das LUCAS-Team regelhaft kontaktiert, um die Bindung aktiv aufrechtzuerhalten und um Adressänderungen, den Eintritt von Pflegebedürftigkeit sowie Todesfälle vollständig und ereignisnah zu erfassen.

- Tab. 2 stellt eine Auswahl von gesundheitsbezogenen Merkmalen (Brückenvariablen) dar, die in den LUCASWellen sowie in den damit verglichenen Querschnittbefragungen identisch erfasst wurden. Ausnahmen betrafen zwei Fragen mit leicht abweichenden Antwortkategorien: Erstens wurde in den anonymen Befragungen nur nach Monat und Geburtsjahr gefragt (Datenschutzauflage). Für die Altersvergleiche mit den Geburtstagen aller Teilnehmenden der LUCAS-Kohorte wurde jeweils der15. Tag des Geburtsmonats angenommen. Zweitens erfolgte die Messung der selbstempfundenen Gesundheit in der Hamburger Befragung im Jahr 2009 mittels 5-stufiger Likert-Skala unterschiedlich zu der auch gebräuchlichen 4-stufigen Skala, die in allen anderen Befragungen zum Einsatz kam, sodass ein verlässlicher Vergleich in diesem Fall unmöglich war.

In der Gegenüberstellung ist die Mehrzahl der in - Tab. 2 zusammengefassten Merkmale gut vergleichbar, wenn Abweichungen um höchstens $5 \%$ als akzeptabel einstuft werden. Die soziodemografischen Variablen der LUCAS-Kohorte sind durchweg sehr gut vergleichbar mit allen drei repräsentativen Befragungen. Lediglich die Hamburger Befragung aus dem Jahr 2009 enthielt im Vergleich mehr Alleinlebende und bzgl. Bildung bestätigt sich [36], dass bei anonymen Querschnittbefragungen teilnehmende Personen einen höheren Schulabschluss besaßen als in der LUCAS-Kohorte, die über ihre Hausarztpraxen rekrutiert wurde (•Tab. 2).

Die Daten zu Mobilität oder Gesundheit boten den Probanden mehr Interpretationsraum (Bsp. Nutzung von Verkehrsmitteln). Die LUCAS-Teilnehmenden nutzten das Auto häufiger als die Befragten in Eimsbüttel (2007 und 2012) und den öffentlichen Personennahverkehr seltener als die Befragten in Eimsbüttel im Jahr 2007, jedoch etwas häufiger als die Befragten in Hamburg im Jahr 2009. Stürze und Sturzangst im Alter haben nicht nur Bezug zum Aktionsradius in der Stadt [24], sondern wegen multidimensionaler Ursachen auch zur körperlichen Funktion und Gesundheit. Verglichen mit den beiden Eimsbütteler Befragungen waren die LUCAS-Teilnehmenden jeweils im Jahrvor der Befragung seltener gestürzt und äußerten auch seltener Sturzangst. Die deutlich seltenere Angabe von Sturzangst und Stürzen in der Befragung in Hamburg (2009) korrespondierte mit den Angaben zu Gesundheit sowie Selbstständigkeit bei Alltagsaktivitäten: Durchweg mehr Personen gaben an, selbstständig und ohne Schwierigkeiten baden oder duschen zu können, 500 Meter zu Fuß zu gehen und leichte Einkäufe zu erledigen. Auch Sehen und Hören wurde häufiger als ausgezeichnet bzw. gut bezeichnet (•Tab. 2).
Anscheinend fühlten sich von der Telefonbefragung (2009) eher gesündere Personen mit höherem Schulabschluss angesprochen; dies möglicherweise, da diese auch größere Anforderungen als eine schriftliche Befragung stellt, bei deren Beantwortung jemand helfen kann. Der Vergleich der Resultate der Befragung in Hamburg (2009) und der LUCAS-Kohorte (neun Jahre nach Rekrutierung über Hausarztpraxen) legt zudem den Schluss nahe, dass vertrauensvoll gewachsene Beziehungen zur Hausarztpraxis, zum Studienzentrum und dessen kontinuierliche Kontaktpflege es auch vergleichsweise weniger Gesunden erleichtert haben könnte, Studienteilnehmende $\mathrm{zu}$ bleiben und sich weiter an den regelmäßigen Befragungen zu beteiligen (Seh- und Höreinschränkungen sowie alltagsrelevante Beeinträchtigungen waren in der LUCASKohorte häufiger, • Tab. 2).

Die Angaben zum selbst eingeschätzten sehr guten oder guten allgemeinen Gesundheitszustand (bei identischer Likert-Skala) sind fast identisch zwischen LUCAS-Welle 2 und der Befragung in Eimsbüttel (2007), vier Jahre später jedoch positiver in LUCAS-Welle 4 als in der Befragung in Eimsbüttel (2012). Die Antworten zwischen der Befragung in Hamburg (2009) und LUCAS-Welle 3 sind wegen unterschiedlicher Ausprägungen der Antwortkategorien nur eingeschränkt vergleichbar. Auch die Fähigkeit, ein Ehrenamt auszuüben, hat Bezug zur eigenen Gesundheit inkl. körperlicher und geistiger Mobilität, was etwas weniger häufig von LUCAS-Teilnehmenden angegeben wurde. Ein Grund dafür mag sein, dass Teilnehmende der LUCAS-Kohorte aufgrund ihrer Möglichkeiten zur Teilnahme an Gesundheitsberatung den Begriff „freiwilliges Engagement" enger interpretierten (• Tab. 2).

Die vergleichsweise hohe Inanspruchnahme medizinischer Vorsorgemaßnahmen in der LUCAS-Kohorte (z. B. Grippeschutzimpfung, zahnärztliche Kontrollen) lässt sich zurückführen auf die Maßnahmen der aktiven Gesundheitsförderung im Alter [15, 24-26] und einen 11 Jahre lang geführten präventivmedizinischen hausärztlichen Qualitätszirkel (- Tab. 2). 


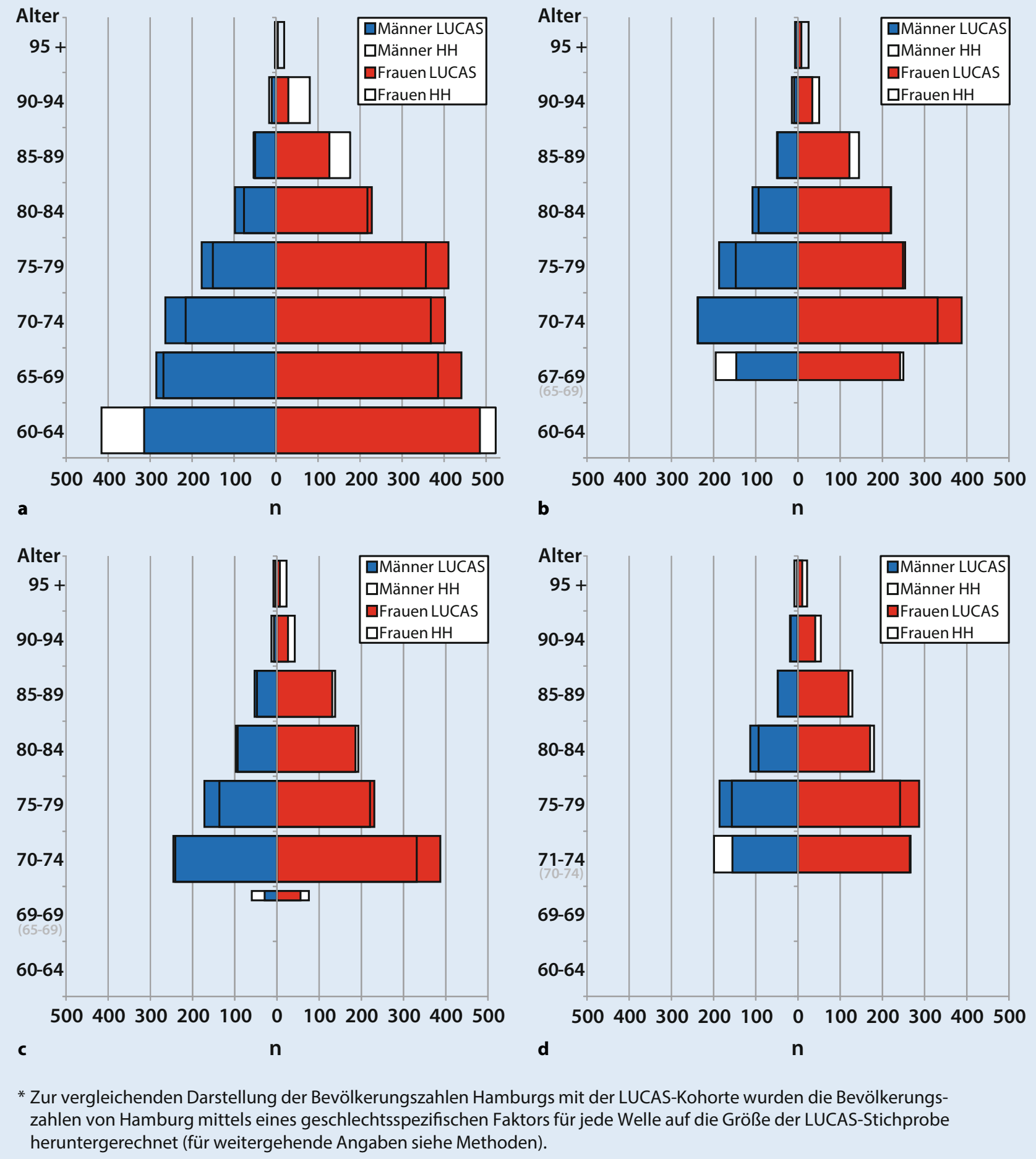

Abb. 2 ム Bevölkerungspyramiden der LUCAS-Teilnehmenden versus der Hamburger Bevölkerung zum 31.12.2000, 31.12.2007, 31.12.2009, 31.12.2011*. Zu Rekrutierung (a) ist die LUCAS-Kohorte in der untersten Altersklasse vergleichsweise klein, da die Rekrutierung nicht das gesamte Vergleichsjahr 2000 durchgeführt wurde, sondern erst ab Oktober 2000 begann. Zu 2007 (b), 2009 (c) und 2011 (d) können aufgrund der jeweiligen Mindestalter in der LUCAS-Kohorte von 67, 69 respektive 71 Jahren keine kompletten 5-Jahres-Altersklassen dargestellt werden 


\section{Exkurs zu anderen Studien epidemiologischer Alterns- forschung in Deutschland}

In einem Workshop der Deutschen Gesellschaft für Epidemiologie wurden Möglichkeiten und Grenzen einer Auswahl zugänglicher Datensätze (public use files) vorhandener Längsschnittund Querschnitterhebungen für die Alternsforschung in Deutschland diskutiert. Die dort vorgestellten Datenquellen - u. a. Deutscher Alterssurvey (DEAS), Studie zur Gesundheit Erwachsener in Deutschland (DEGS), Kooperative Gesundheitsforschung in der Region Augsburg (KORA-Age), Sozio-oekonomisches Panel (SOEP), Survey of Health, Ageing and Retirement in Europe (SHARE) - unterscheiden sich sowohl hinsichtlich Zielsetzung, Art und Region der Erhebung als auch Stichprobengröße und Altersbereich, Responsequote sowie Repräsentativität [16].

Trotz der damals festgestellten Heterogenität wird folgend der Versuch unternommen, publizierte Angaben aus diesen Studien mit LUCAS-Daten zu vergleichen. Dafür wurde jeweils die zeitlich passende LUCAS-Welle ausgewählt, der LUCAS-Datensatz bestmöglich angepasst und analysiert, um ihn mit den Angaben der anderen Kohorten zu vergleichen ( $\bullet$ Tab. 3a-e).

Mit einer Ausnahme konnte für jede der bundesweiten Studien (DEGS, DEAS, SHARE), mindestens ein publizierter Vergleichswert identifiziert werden. Vergleiche mit SOEP waren nicht möglich, da die veröffentlichten Resultate jeweils alle Haushaltsmitglieder ab 17 Jahren umfassten [37]. Vergleiche zwischen LUCAS und DEAS zeigten ähnliche prozentuale Anteile deutscher Staatsangehörigkeit sowie ehrenamtlich Tätiger, allerdings nicht für den Bildungsstand. Aufgrund der unteren und oberen Altersgrenzen in DEAS (40-85 Jahre) sind solche Vergleiche allerdings nur eingeschränkt möglich. Vergleiche der subjektiv empfundenen Gesundheit sind wegen unterschiedlicher Antwortkategorien nicht möglich [38]. Die Antwortkategorien zur subjektiven Gesundheit ähneln sich in SHARE Deutschland und LUCAS; deren Zusam- menfassungen zeigten fast identische Prozentanteile. Allerdings ist zumindest die untere Altersgrenzenbelegung bei SHARE (50 Jahre) zu berücksichtigen, die auch den geringeren Anteil der Personen mit Krankenhausaufenthalt in SHARE bedingen dürfte. LUCAS-Daten zeigten höhere Anteile der Personen mit Zahnkontrolle im Unterschied zu SHARE [39]. Ursache dafür könnten die im Rahmen von LUCAS angebotenen gesundheitsfördernden Interventionen sein. Die Anteile ehrenamtlich Tätiger liegen in LUCAS zwischen denen der zwei deutschen SHARE-Vergleichswellen $[39,40]$. Der, verglichen mit LUCAS, um $10 \%$ geringere Frauenanteil in der höchsten DEGS-Altersklasse (70 bis 79 Jahre) [9] legt die Frage zur Repräsentativität im hohen Alter nahe. Gleiches gilt für den Frauenanteil in der höchsten Altersklasse (61-75 Jahre) in der Epidemiologischen Studie zu Chancen der Verhütung, Früherkennung und optimierten Therapie chronischer Erkrankungen in der älteren Bevölkerung (ESTHER) im Bundesland Saarland [41]. Am ehesten vergleichbar bezüglich der publizierten Daten sind die beiden regionalen Kohorten LUCAS und KORAAge mit der Einschränkung, dass LUCAS-Probanden zwei Jahre älter sind. KORA-Age Daten zeigen eine ähnliche Geschlechterverteilung in der Region Augsburg, mit etwas geringeren Anteilen höher Gebildeter und vergleichbaren Gesundheitsdaten [42].

\section{Diskussion}

Abweichend von üblichen Stichprobenziehungen über Einwohnermelderegister wurden LUCAS-Teilnehmende über ihre Hausarztpraxen rekrutiert. Seit Beginn der Langzeitkohortenstudie LUCAS wird deshalb die Repräsentativität der Ergebnisse überprüft. Die hier verwendete Definition des Begriffs „Repräsentativität“ entspricht der wechselseitigen Vergleichbarkeit verschiedener Datenquellen bezüglich definierter Charakteristika.

Bekanntermaßen treten Nichtteilnahme bzw. reduzierte Erreichbarkeit sowohl in der amtlichen als auch in der LUCAS-Befragung auf, sodass es nicht a priori klar ist, welche Angaben nun verlässlicher sind. Vielfach wird eine hohe Verlässlichkeit der amtlichen Befragung angenommen: Bürger sind in der Regel bereit, staatlichen Aufforderungen nachzukommen, d.h. ihnen vorgelegte Fragen zu beantworten. Aufgrund der gewählten Erhebungsmethoden darf angenommen werden, dass die Ergebnisse der drei Querschnitterhebungen trotz niedriger, aber bei anonymen Befragungen zu erwartenden Ausschöpfungsquoten zwischen 20 und $30 \%$ repräsentativ sind bezüglich Alter, Geschlecht und Wohnregion (Befragung Eimsbüttel 2012), Alter und Geschlecht (Befragung Eimsbüttel 2007) sowie Alter (Befragung Hamburg 2009). Auch der Abgleich mit den Daten der Non-Responder in der Befragung in Hamburg (2009) lässt auf eine valide Datengrundlage in Bezug auf die ältere Bevölkerung schließen [33].

Die Analysen können zur Beantwortung der vier Forschungsfragen zur Repräsentativität und Vergleichbarkeit der LUCAS-Daten und Ergebnisse dienen.

1.) Die Grafiken und Analysen erlauben den Schluss, dass die über Hausarztpraxen rekrutierten Teilnehmenden der LUCAS-Langzeitkohorte im Verlauf gut vergleichbar sind mit der älteren Hamburger Gesamtbevölkerung auf Basis der amtlichen Statistik. Männer der Altersgruppe 60-64 Jahre in 2000/01 (um 100 Personen) sind vermutlich untervertreten, weil diese, noch im Arbeitsprozess, seltener eine Hausarztpraxis aufsuchen. Weiter findet sich bei den 90 - bis 94 -jährigen Frauen in 2000/01 eine geringe (um 50 Personen), aber anteilig wichtige Unterrepräsentation, was wahrscheinlich auf die LUCAS-Einschlussbedingung „selbstständig, nicht pflegebedürftig“ zurückzuführen ist. Geringe Überrepräsentationen finden sich für beide Geschlechter in der Alterskategorie 65 bis 84 Jahre. In den späteren Vergleichen 2007/08, 2009/10 und 2011/12 sind diese Unter- bzw. Überrepräsentationen allerdings deutlich reduziert. Zur Hochrechnung der Häufigkeit von gesundheitlichen Angaben (deutlich geschlechts- bzw. altersabhängig) auf andere Populationen sollten deshalb gewichtete Kombinationen von altersund geschlechtsspezifischen LUCASAnteilen verwendet werden. 


\section{Originalien und Übersichten}

Tab. 3 Vergleiche zwischen identischen bzw. ähnlichen Fragestellungen der LUCAS-Kohortenstudie und publizierten Daten anderer Alters-Kohortenstudien (alphabetische Sortierung) deutschlandweit (DEGS, DEAS, SHARE) und regional (ESTHER, KORA-Age) a LUCAS (Hamburg) $2007 / 08$ vs. DEGS (Deutschland) 2008/11; b LUCAS (Hamburg) 2007/08 vs. DEAS (Deutschland) 2008; c LUCAS (Hamburg) 2007/08 vs. SHARE (Deutschland) 2004/05 sowie SHARE (Deutschland) 2006/07; d LUCAS (Hamburg) 2000/01 vs. ESTHER (Saarland) 2000/02; e LUCAS (Hamburg) 2007/08 vs. KORA-Age (Region Augsburg) $2006 / 07$

\section{a}

Vergleichbares Merkmal auf Basis publizierter DEGS Daten (in [9]; Tab. 1, S. 726)

LUCAS 2007/08

70-79 Jahre $n=$ 1067 $642 / 1067(60,2)$

Frauen $70-79$ Jahre: $n(\%)$ b

Vergleichbare Merkmale auf Basis publizierter DEAS Daten (Basisstichprobe 2008, gewichtete Angaben) in [38]

Deutsche: in \%

LUCAS 2007/08

67 Jahre und älter

$n=2012$

96,9

Höherer Schulabschluss: in \% $\quad 39,9 \quad 57,9$

Höherer Schulabschluss: in \% $\quad 39,9 \quad 57,9$

Vergleichbare Merkmale auf Basis publizierter DEAS Daten (Basisstichprobe 2008, gewichtete Angaben) in [38]

\begin{tabular}{|c|c|c|c|}
\hline Ehrenamt in \% & 11,0 & 12,0 & $\begin{array}{l}\text { Zusätzlich eingeschränkt vergleichbar aufgrund Fragestellung: } \\
\text { LUCAS: Ehrenamtliches Engagement } \\
\text { DEAS: Außerberufliche gesellschaftliche Partizipation als ehrenamt- } \\
\text { liches Engagement und Bildung als lebenslanges Lernen (vgl. [38]; } \\
\text { Abb. 5-7, S. 134) }\end{array}$ \\
\hline $\begin{array}{l}\text { Subjektive Gesundheit sehr } \\
\text { gut/gut in \% }\end{array}$ & 59,6 & 40,0 & \multirow{3}{*}{$\begin{array}{l}\text { Zusätzlich eingeschränkt vergleichbar aufgrund Antwortkategorien } \\
\text { und deren Zusammenfassung in DEAS-Ergebnisdarstellung in nur } \\
3 \text { Gruppen: } \\
\text { LUCAS: 4er-Skala: sehr gut, gut, mäßig, schlecht } \\
\text { DEAS: 5er-Skala: sehr gut, gut, mittel, schlecht, sehr schlecht (vgl. [38]; } \\
\text { Abb. 4-7, S. 105) }\end{array}$} \\
\hline $\begin{array}{l}\text { Subjektive Gesundheit mittel in } \\
\%\end{array}$ & 36,3 & 43,0 & \\
\hline $\begin{array}{l}\text { Subjektive Gesundheit sehr } \\
\text { schlecht/schlecht in \% }\end{array}$ & 4,1 & 18,0 & \\
\hline \multicolumn{4}{|l|}{ c } \\
\hline \multirow[t]{2}{*}{$\begin{array}{l}\text { Vergleichbares Merkmal auf Ba- } \\
\text { sis publizierter SHARE Deutsch- } \\
\text { land (D) Daten in [39] }\end{array}$} & $\begin{array}{l}\text { LUCAS 2007/08 } \\
\text { Alle Befragten 67+ } \\
\text { Jahre } n=2012\end{array}$ & $\begin{array}{l}\text { SHARE (D) 2004/05 } \\
\text { Alle Befragten } 50+ \\
\text { Jahre } n=2281\end{array}$ & $\begin{array}{l}\text { Einschränkungen } \\
\text { 1. Andere Altersvergleiche waren nicht möglich, da bei SHARE Auswer- } \\
\text { tungen immer } 50 \text { Jahre und älter (vgl. }[39,40])\end{array}$ \\
\hline & & & $\begin{array}{l}\text { 2. Zeitdifferenz beträgt } 3 \text { Jahre zwischen verglichenen Befragungs-Wel- } \\
\text { len }\end{array}$ \\
\hline $\begin{array}{l}\text { Subjektive Gesundheit (exzel- } \\
\text { lent)/sehr gut/gut in \% }\end{array}$ & 61,0 & 59,4 & \multirow{2}{*}{$\begin{array}{l}\text { Zusätzlich eingeschränkt vergleichbar aufgrund Antwortkategorien: } \\
\text { LUCAS: 4er-Skala: sehr gut, gut, mäßig, schlecht } \\
\text { SHARE: 5er-Skala: exzellent, sehr gut, gut, mäßig, schlecht (vgl. [39]; } \\
\text { Tab. } 3 \text { A.11, S. 154) }\end{array}$} \\
\hline $\begin{array}{l}\text { Subjektive Gesundheit mäßig/ } \\
\text { schlecht in \% }\end{array}$ & 39,0 & 40,6 & \\
\hline $\begin{array}{l}\text { Zahnkontrolle in den letzten } \\
12 \text { Monaten in \% }\end{array}$ & 84,7 & 72,1 & (Vgl. [39]; Tab. 3 A.26, S. 160) \\
\hline $\begin{array}{l}\text { Kein Krankenhausaufenthalt in } \\
\text { den letzten } 12 \text { Monaten in \% }\end{array}$ & 71,0 & 83,1 & (Vgl. [39]; Tab. 3 A.28, S. 160) \\
\hline \multirow[t]{2}{*}{ Ehrenamt in \% } & 11,3 & 10,1 & $\begin{array}{l}\text { Zusätzlich eingeschränkt vergleichbar aufgrund Fragestellung (vgl. [40]; } \\
\text { Tab. 1, S. 241) }\end{array}$ \\
\hline & $\begin{array}{l}\text { LUCAS 2007/08 } \\
\text { Alle Befragten } 67+ \\
\text { Jahre } n=2012\end{array}$ & $\begin{array}{l}\text { SHARE (D) } 2006 / 07 \\
\text { Alle Befragten } 50+ \\
\text { Jahre } n=\mathrm{k} . \text {.A. }\end{array}$ & $\begin{array}{l}\text { Andere Altersvergleiche waren nicht möglich, da bei SHARE Auswer- } \\
\text { tungen immer } 50 \text { Jahre und älter (vgl. }[39,40])\end{array}$ \\
\hline Ehrenamt \% & 11,3 & 13,1 & $\begin{array}{l}\text { Zusätzlich eingeschränkt vergleichbar aufgrund Fragestellung (vgl. [40]; } \\
\text { Tab. 1, S. 241) }\end{array}$ \\
\hline
\end{tabular}

LUCAS 2007/08 DEAS 2008

$67+$ Jahre $n=2012 \quad 70-85$ Jahre $n=$ k.A.
DEGS $2008 / 11$

$70-79$ Jahre $n=$ $556 / 1110(50,1)$

\section{DEAS 2008 Einschränkungen}

6205

96,2

12,0
Einschränkungen Altersvergleiche in höheren Altersklassen waren nicht möglich, da bei DEGS obere Altersgrenze bei 79 Jahren liegt. (vgl. [9]; Abb. 1, S. 724)
Zusätzlich eingeschränkt vergleichbar aufgrund Fragestellung: LUCAS: Geburtsort Deutschland DEAS: Staatsangehörigkeit deutsch (vgl. [38]; Tab. 2-5, S. 45)

Zusätzlich eingeschränkt vergleichbar aufgrund Antwortkategorien: LUCAS: Abitur/Matura/Fachhochschulreife, Realschule/Lyzeum DEAS: Abitur/Fachhochschulreife, Realschule/Mittlere Reife, Polytechn. Oberschule (vgl. [38]; Tab. 2-5, S. 45)

Eingeschränkt vergleichbar aufgrund Alter (s. oben)

Zusätzlich eingeschränkt vergleichbar aufgrund Antwortkategorien 3 Gruppen: DEAS: 5er-Skala: sehr gut, gut, mittel, schlecht, sehr schlecht (vgl. [38]; Abb. 4-7, S. 105) Tab. 1, S. 241) 
Tab. 3 Vergleiche zwischen identischen bzw. ähnlichen Fragestellungen der LUCAS-Kohortenstudie und publizierten Daten anderer Alters-Kohortenstudien (alphabetische Sortierung) deutschlandweit (DEGS, DEAS, SHARE) und regional (ESTHER, KORA-Age) a LUCAS (Hamburg) 2007/08 vs. DEGS (Deutschland) 2008/11; b LUCAS (Hamburg) 2007/08 vs. DEAS (Deutschland) 2008; c LUCAS (Hamburg) 2007/08 vs. SHARE (Deutschland) 2004/05 sowie SHARE (Deutschland) 2006/07; d LUCAS (Hamburg) 2000/01 vs. ESTHER (Saarland) 2000/02; e LUCAS (Hamburg) 2007/08 vs. KORA-Age (Region Augsburg) $2006 / 07$ (Fortsetzung)

\begin{tabular}{|c|c|c|c|}
\hline $\begin{array}{l}\text { Vergleichbares Merkmal auf Ba- } \\
\text { sis publizierter ESTHER Daten } \\
\text { (in [41]; Tab. 1, S. 37) }\end{array}$ & $\begin{array}{l}\text { LUCAS 2000/01 } \\
61-75 \text { Jahre } n= \\
2247\end{array}$ & $\begin{array}{l}\text { ESTHER 2000/02 } \\
61-75 \text { Jahre } n= \\
3600\end{array}$ & $\begin{array}{l}\text { Einschränkungen } \\
\text { Eingeschränkt vergleichbar, da die Auswahl dieser Altersklasse 61-75 } \\
\text { (vgl. [41]; Tab. 1) aus unterschiedlichen Stichproben-Grundgesamthei- } \\
\text { ten stammt: } \\
\text { LUCAS Alter zu Rekrutierung 2000: } 60 \text { Jahre und älter } \\
\text { ESTHER Alter zu Rekrutierung 2000: 50-74 Jahre }\end{array}$ \\
\hline Frauen 61-75 Jahre: $n$ (\%) & $1372 / 2247(61,1)$ & $1878 / 3600(52,2)$ & \\
\hline \multicolumn{4}{|l|}{ e } \\
\hline $\begin{array}{l}\text { Vergleichbare Merkmale auf } \\
\text { Basis publizierter KORA-Age } \\
\text { Daten (in [42]; Tab. 2, S. 47) }\end{array}$ & $\begin{array}{l}\text { LUCAS 2007/08 } \\
67+\text { Jahre } n=2012\end{array}$ & $\begin{array}{l}\text { KORA-Age 2008/09 } \\
65+\text { Jahre } n=4565\end{array}$ & $\begin{array}{l}\text { Einschränkungen Allgemein: Eingeschränkt vergleichbar, da die LUCAS } \\
\text { Kohorte zum Vergleich der beiden Befragungs-Wellen zwei Jahre älter } \\
\text { war }\end{array}$ \\
\hline $\begin{array}{l}\text { Alter zur Befragung (in J.): Mit- } \\
\text { telwert (Minimum-Maximum) }\end{array}$ & $76,2(67,0-99,7)$ & 73,9 (k. A.) & \\
\hline Frauen: $n(\%)$ & $1269 / 2012(63,1)$ & $2367 / 4565(51,9)$ & \\
\hline $\begin{array}{l}\text { Höherer Schulabschluss Abitur/ } \\
\text { Realschule: } n(\%)\end{array}$ & $741 / 1857(39,9)$ & $1335 / 4563(29,3)$ & $\begin{array}{l}\text { Zusätzlich eingeschränkt vergleichbar aufgrund Antwortkategorien: } \\
\text { LUCAS: Abitur/Matura/Fachhochschulreife, Realschule/Lyzeum } \\
\text { KORA-Age: Abitur/Fachabitur/Hochschulreife, Mittlere Reife/Realschule } \\
\text { (vgl. [42]; Tab. 2, S. 47) }\end{array}$ \\
\hline $\begin{array}{l}\text { BMI: Mittelwert (Mini- } \\
\text { mum-Maximum) }\end{array}$ & $26,4(15-49)$ & $27,45(-)$ & \\
\hline Diabetes: $n(\%)$ & $322 / 2000(16,1)$ & $768 / 4565(17,5)$ & \\
\hline $\begin{array}{l}\text { Koronare Herzerkrankung: } n \\
\text { (\%) }\end{array}$ & $437 / 1957(22,3)$ & $1034 / 4565(22,7)$ & $\begin{array}{l}\text { Zusätzlich eingeschränkt vergleichbar aufgrund Fragestellung: } \\
\text { LUCAS: Angina pectoris, Erkrankung der Herzkranzgefäße, Herzinfarkt } \\
\text { KORA-Age: Koronare Herzerkrankung, Bypassoperation, Myokardinfarkt } \\
\text { (vgl. [42]; Tab. 2, S. 47) }\end{array}$ \\
\hline
\end{tabular}

2.) Abweichungen von jeweils über $5 \%$ finden sich bei 13 der insgesamt 28 in - Tab. 2 aufgeführten Merkmale. Deutliche Abweichungen bei soziodemografischen Merkmalen treten nur bei zwei von sieben Merkmalen auf. Beim „höheren Schulabschluss“ zeigt sich, dass nur die weniger Deutungsspielraum erlaubende Frage nach einer abgeschlossenen akademischen Ausbildung verlässliche Antworten liefert. Die Abweichung bei „allein lebend“ zeigt sich lediglich bei der Hamburger Erhebung 2009, was vermutlich in der Erhebungsart begründet ist. Die meisten nennenswerten Abweichungen betrafen Mobilitätsfragen (5/6), die nicht direkt verifizierbare Tatsachen, sondern Aussagen über kürzer oder länger zurückliegende Zeiträume betreffen, was deren Zuverlässigkeit beeinträchtigt. Die Antworten auf Fragen zur Gesundheit und Selbstständigkeit zeigen bei vier von 13 Merkmalen Abweichungen von über $5 \%$. Die überwiegend gute Übereinstimmung mag daran liegen, dass hier - ähnlich wie bei soziodemografischen Merkmalen - Fragen nach Fakten gestellt wurden wie z. B. Krankenhausaufenthalt, Pflegestufe, Hilfsmittel zur Fortbewegung, Selbstständigkeit beim Baden, dauerhafte Schmerzen, Anzahl Medikamente, BMI oder Zigarettenkonsum. Daraus kann gefolgert werden, dass die ermittelten soziodemografischen Merkmale in verschiedenen LUCASBefragungswellen verlässlich sind. Die Antworten auf Faktenfragen zur Gesundheit und Selbstständigkeit weisen lediglich geringe Unterschiede zwischen den Studien auf, sind also als verlässlich einzustufen und gut vergleichbar. Angaben zu Mobilität und Bildung bieten jedoch mehr Interpretationsspielraum bei der Beantwortung, was deren Vergleichbarkeit beeinträchtigt.

3.) Registerbasierte Erhebungen mit Anspruch auf Repräsentativität liefern vergleichbare Ergebnisse zu LUCAS, wenn sich die Befragungsmethode sowie die erhobenen Variablen und deren
Ausprägungen entsprechen und wenn die Variablen direkt verifizierbare Fakten betreffen. Es fanden sich keine signifikanten Unterschiede bezüglich soziodemografischer Kennzahlen (Alter, Geschlecht, Staatsangehörigkeit), die üblicherweise für Repräsentativitätsgewichtungen herangezogenen werden. Unterschiedliche Werte bei der Selbsteinschätzung des Gesundheitszustandes sind zumindest teilweise auf die unterschiedlichen Anzahlen und Ausprägungen der Antwortkategorien (4er- versus 5er-LikertSkala) zurückzuführen.

4.) Trotz unterschiedlicher Rekrutierungsstrategien über Hausarztpraxen in LUCAS (nicht repräsentativ bezüglich Arztpraxen, komplette Erfassung aller älteren Personen, niedrige Ausfallrate) und das Einwohnermeldeamt in den Hamburger Querschnittbefragungen (repräsentative Auswahl, anonyme Befragung, hohe Ausfallrate), die auch unterschiedliche Stichproben erreichten, zeigen sich überwiegend hohe Übereinstimmungen 
der Antworten zu gesundheitsrelevanten Fragen (Krankenhausaufenthalte, Pflegestufe). Signifikante Abweichungen in der LUCAS-Kohorte bezüglich höherer Inanspruchnahme von Maßnahmen der Gesundheitsvorsorge (Zahnkontrollen, Grippeschutzimpfung) sowie Sturzprävention (weniger Stürze und Sturzangst) sind sehr wahrscheinlich beeinflusst durch gesundheitsfördernde Interventionen für die LUCAS-Teilnehmenden und den präventivmedizinischen Qualitätszirkel für die LUCAS-Hausarztpraxen.

Folgende limitierende Faktoren sind für diese Vergleiche $\mathrm{zu}$ berücksichtigen: Sämtliche LUCAS-Befragungswellen wurden über neun Monate, die Querschnittbefragungen hingegen während zwei bis fünf Monaten durchgeführt. Die Angaben aus der Hamburger Bevölkerungsstatistik bezogen sich jeweils auf den 31. Dezember eines Jahres, die Daten aus den LUCAS-Wellen jedoch auf die Periode Oktober desselben Jahres bis zum folgenden Juni. Für die Vergleiche der Wellen 3 und 4 musste mit gewichteten Datensätzen der Querschnittbefragungen operiert werden, da die ungewichteten Daten nicht die Verhältnisse in der Zielpopulation der Querschnittstudien ergeben hätten (siehe Datenanalyse und Ergebnisdarstellung). Ein eventuell durch die Art der Befragung bedingter Selektionsbias muss beachtet werden. So erfolgte die Hamburger Querschnittbefragung im Jahr 2009 nicht schriftlich, sondern über eine standardisierte Telefonbefragung; aber nur jede zweite ältere Person verfügte über einen Telefonbucheintrag [33]. Demgegenüber erfasst LUCAS lediglich Personen mit Hausarzt (fast jede ältere Person [43]). Zu beachten ist zudem, dass die relativen Stichprobengrößen zwischen der LUCAS-Kohorte und den Querschnittstudien variieren von 1:2 (Eimsbüttel-Befragung 2012) bis 3:1 (Befragung Hamburg 2009), vgl.

\section{- Tab. 2.}

Schließlich sind allgemeine Limitationen zu bedenken, wie die Verlässlichkeit von Selbstangaben [44], die Nichtteilnahme [45] und die Verluste im Verlauf von Langzeitstudien [46]. Für die LUCAS-Langzeitkohorte wurden Ver- lässlichkeit schriftlicher Angaben mittels multidimensionaler Performance-Tests validiert [14] und Nichtteilnahme bei Rekrutierung [27] sowie die Verlustraten während der ersten 10 Jahre erhoben und analysiert [31].

Mit Blickauf die Erhebung repräsentativer deutschlandweiter Daten zu älteren und hochaltrigen Menschen sowie deren Überprüfung auf Repräsentativität im Langzeitverlauf könnten - basierend auf den LUCAS-Erfahrungen - Hausarztpraxen möglicherweise gewinnbringend einbezogen werden. Zudem könnte der Funktionsstatus der Probanden (z. B. über kurzes Selbstausfüllerscreening) genutzt werden, um anschließende Untersuchungen und Performancetestungen zielgruppenspezifisch anbieten zu können: Für rüstige ältere Menschen im Studienzentrum, für fraile Personen in der Hausarztpraxis oder im Hausbesuch. In den Untersuchungsregionen der bundesweiten Studienzentren könnten zusätzlich $\mathrm{zu}$ den repetitiven Befragungswellen der Langzeitkohorte zeitlich synchronisiert repräsentative Stichproben für Querschnittbefragungen gezogen werden. Über sog. Brückenvariablen (identische Fragestellungen und Antwortkategorien) könnte so die Vergleichbarkeit auch im Langzeitverlauf überprüft und ggf. angepasst werden.

\section{Fazit und Ausblick}

Jeder fünfte in Deutschland lebende Einwohner ist mindestens 65 Jahre alt, und fast jeder Zehnte hat bereits das 75 . Lebensjahr erreicht oder überschritten [37]. Die Anpassung der Gesundheitsund Versorgungssysteme sollte Früherkennungskonzepte für vulnerable und gebrechliche ältere Menschen (Frailty) berücksichtigen, um dem Eintritt unerwünschter Folgen wie Funktionsverlusten (disability) und Pflegebedürftigkeit entgegenzutreten [47]. Schlüsselkomponenten für Präventions- und Therapieplanung sind ein multidimensionales Assessment und transsektorale Versorgung, wobei Hausarztpraxen eine zentrale Rolle zukommt [39].

Derzeit fehlen jedoch in Deutschland repräsentative Daten zu hochaltrigen Menschen. Gründe dafür könnten u. a. befürchtete Nichtteilnahme und/ oder hohe Abbruchraten im Verlauf sein [11]. In der seit 2000 in Hamburg kontinuierlich fortgeführten LUCASLangzeitkohortenstudie ohne obere Altersgrenze liegt die mittlere Studienabbruchrate (ohne Verstorbene) bisher unverändert unter $3 \%$ pro Jahr. Die oberen Altersgrenzen in sehr wichtigen deutschlandweiten Surveys liegen bei 69 Jahren (Nationale Kohorte [48]), bei 79 Jahren (Studie zur Gesundheit Erwachsener in Deutschland, DEGS [49]) und bei 85 Jahren (Deutschen Alterssurvey, DEAS [38]). Abhängig von dieser Altersbegrenzung sind zwischen 3 bis $16 \%$ der Gesamtbevölkerung [50] von einer Studienteilnahme ausgeschlossen. Der Ausschluss hochaltriger Menschen von gesundheitspolitisch relevanten Studien ist nicht zu rechtfertigen [4, 47].

Es wurde gezeigt, dass die in der LUCAS-Langzeitkohortenstudie während 12 Jahren generierten Ergebnisse zur Gesundheit älterer Menschen in wesentlichen Aspekten vergleichbar sind mit denen repräsentativen Querschnitterhebungen, die im selben Raum erhoben wurden. Dies betraf sowohl soziodemografische Angaben als auch die Inanspruchnahme von Gesundheitsleistungen, wie z.B. Krankenhausaufenthalte oder Hilfsmittelnutzung, sowie weitere selbstberichtete Angaben zur Gesundheit. Diese Informationen können zur frühzeigen Identifikation von Frailty, Vorsorge- und Behandlungsbedarf sowie auch zur Planung und Durchführung zielgruppenspezifischer Maßnahmen herangezogen werden (z. B. [51]).

\section{Korrespondenzadresse}

\section{Dr. rer. nat. U. Dapp}

Albertinen-Haus Zentrum für Geriatrie und Gerontologie, Wissenschaftliche Einrichtung an der Universität Hamburg

Sellhopsweg 18-22, 22459 Hamburg,

Deutschland

ulrike.dapp@albertinen.de

Danksagung. Wir danken den LUCASTeilnehmerinnen und -Teilnehmern, ihren Hausarztpraxen, den älteren Menschen der drei Hamburger Querschnitt-Befragungen, dem Fachamt Einwohnerwesen und dem Datenschutzbeauftragten der Freien und Hansestadt Hamburg sowie dem Medizinischen Dienst der Krankenversicherung Nord, 
ohne die die Datenerhebung nicht möglich gewesen wäre. Die Seniorenbefragungen Eimsbüttel 2007 und 2012 wurden aus Mitteln des Bezirks Eimsbüttel finanziert. Die Seniorenbefragung 2009 „Die Gesundheit älterer Menschen in Hamburg" wurde gefördert vom Bundesministerium für Bildung und Forschung (BMBF LUCAS I: 01ET0709). PRO-AGE und LUCAS wurden gefördert von der Europäischen Kommission (QLK6-CT-1999-02205), dem Bundesministerium für Familie, Senioren, Frauen und Jugend (BMFSFJ), der Robert Bosch Stiftung, der Max und Ingeburg Herz Stiftung sowie dem Bundesministerium für Bildung und Forschung (BMBF LUCAS I: 01ET0708+01ET0709, BMBF LUCAS II: 01ET1002 A, BMBF LUCAS III/PROLONG HEALTH: 01EL1407).

\section{Einhaltung ethischer Richtlinien}

Interessenkonflikt. U. Dapp, M. Dirksen-Fischer, G. Rieger-Ndakorerwa, R. Fertmann, K.-P. Stender, S. Golgert, W. von Renteln-Kruse und C.E. Minder geben an, dass kein Interessenkonflikt besteht.

Dieser Beitrag beinhaltet keine von den Autoren durchgeführten Studien an Menschen oder Tieren.

Open Access. This article is distributed under the terms of the Creative Commons Attribution 4.0 International License (http://creativecommons.org/ licenses/by/4.0/), which permits unrestricted use, distribution, and reproduction in any medium, provided you give appropriate credit to the original author(s) and the source, provide a link to the Creative Commons license, and indicate if changes were made.

\section{Literatur}

1. EuroHealthNet (2012) Healthy and active ageing. (Brussels) www.healthyageing.eu/sites/ www.healthyageing.eu/files/resources/Healthy \%20and\%20Active\%20Ageing.pdf (Erstellt: January 2012). Zugegriffen: 1. Juli 2015

2. Steinberg J, Doblhammer-Reiter G (2010) Demografische Bevölkerungsprognosen. Theoretische Grundlagen, Annahmen und Vorhersagesicherheit. Bundesgesundheitsbl Gesundheitsforsch Gesundheitsschutz 53:393-403

3. Böhm K, Tesch-Römer C et al (Hrsg) (2009) Gesundheit und Krankheit im Alter. Robert KochInstitut, Berlin

4. Clegg A, Young J, lliffe S et al (2013) Frailty in elderly people. Lancet 381:752-762

5. Fried LP, Tangen CM, Walston J et al (2001) Frailty in older adults: evidence for a phenotype. J Gerontol A Biol Med Sci 56(A):M146-M156

6. Rockwood K, Song X, MacKnight C et al (2005) A global clinical measure of fitness and frailty in elderly people. CMAJ 173:489-495

7. De Vries NM, Staal JB, van Ravensberg CD et al (2011) Outcome instruments to measure frailty: a systematic review. Ageing Res Rev 10:104-114

8. Salomon JA, Wang H, Freeman MK et al (2012) Healthy life expectancy for 187 countries, 1990-2010: a systematic analysis for the Global Burden Disease Study 2010. Lancet 380:2144-2162

9. Fuchs J, Busch MA, Gößwald A et al (2013) Körperliche und geistige Funktionsfähigkeit bei Personen im Altervon 65 bis 79 Jahren in Deutschland. Ergebnisse der Studie zur Gesundheit Erwachsener in Deutschland (DEGS1). Bundesgesundheitsbl Gesundheitsforsch Gesundheitsschutz 56:723-732
10. Plass D, Vos T, Scheidt-Nave C et al (2014) Trends in disease burden in Germany - results, implication and limitations of the Global Burden of Disease Study. Dtsch Arztebl Int 111:629-638

11. Guralnik JM, Kritchevsky SB (2010) Translating research to promote healthy aging: the complementary role of longitudinal studies and clinical trials. J Am Geriatr Soc 58(Suppl 2):S337-S342

12. Dapp U, Fertmann R, Anders J et al (2011) Die Longitudinal-Urban-Cohort-Ageing-Studie (LUCAS).Z Gerontol Geriatr 44(Suppl 2):55-71

13. Dapp U, Minder C, Anders J et al (2014) Longterm prediction of changes in health status, frailty, nursing care and mortality in community-dwelling senior citizens - results from the Longitudinal Urban Cohort Ageing Study (LUCAS). BMC Geriatr 14:141 (http://www.biomedcentral.com/1471 2318/14/141).Zugegriffen: 1. Juli 2015

14. Anders J, Pröfener F, Dapp U et al (2012) Grauzonen von Gesundheit und Handlungsfähigkeit. Erfahrungen und Aufschlüsselung durch erweiterte Assessments in der Longitudinalen Urbanen Cohorten-Alters-Studie (LUCAS). Z Gerontol Geriat 45:271-278

15. Dapp U, Anders JA, von Renteln-Kruse W et al (2011) A randomized trial of effects of health risk appraisal combined with group sessions or home visits on preventive behaviors in older adults. J Gerontol A Biol Sci Med Sci 66(A):591-598 (for the PRO-AGEStudy Group)

16. Fuchs J, Grabka M, Gruber S et al (2013) Daten für die epidemiologische Altersforschung. Möglichkeiten und Grenzen vorhandener Datensätze. Ergebnisse des 2. Workshops der Arbeitsgruppe Epidemiologie des Alterns der Deutschen Gesellschaft für Epidemiologie (DGEpi). Bundesgesund heitsbl Gesundheitsforsch Gesundheitsschutz 56:1425-1431

17. Kish L (1965) Survey Sampling. Wiley, New York, S36

18. Statistisches Landesamt der Freien und Hansestadt Hamburg (Hrsg) (2002) Bevölkerung am 31.12.2000 nach Alter und Geschlecht. Fortschreibung auf der Basis der VZ 1987. Statistischer Bericht A I 3 - j/00. Herausgegeben am 24. Januar 2002

19. Statistisches Amt für Hamburg und SchleswigHolstein (Hrsg) (2008) Die Bevölkerung in Hamburg und Schleswig-Holstein 2007 nach Alter und Geschlecht. Fortschreibung auf der Basis der VZ 1987. Statistischer Bericht A I 3 - j/07. Herausgegeben am 21. August 2008

20. Statistisches Amt für Hamburg und SchleswigHolstein (Hrsg) (2010) Die Bevölkerung in Hamburg und Schleswig-Holstein 2009 nach Alter und Geschlecht. Fortschreibung auf der Basis der VZ 1987. Statistischer Bericht A I 3 - j/09. Herausgegeben am 01. September 2010

21. Statistisches Amt für Hamburg und SchleswigHolstein (Hrsg) (2013) DieBevölkerung in Hamburg am 31. Dezember 2011 nach Alter und Geschlecht. Fortschreibung auf der Basis der VZ 1987. Statistischer Bericht AI3 - j/11. Herausgegeben am 02. August 2012

22. Statistisches Amt für Hamburg und SchleswigHolstein(Hrsg) (2014)DieBevölkerung in Hamburg nach Alter und Geschlecht 2011. Fortschreibung auf der Basis des Zensus 2011. Statistischer Bericht Al3-j/11 HH. Herausgegeben am 10. Juli 2014

23. Folstein MF, Folstein SE, KcHugh PR (1975) "Minimental state". A practical method for grading the cognitive state of patients for the clinician. J Psychiatr Res 12:189-198
24. Dapp U (2008) Gesundheitsförderung und Prävention selbständig lebender älterer Menschen. Eine medizinisch-geographische Untersuchung. Kohlhammer, Stuttgart

25. Meier-Baumgartner HP, Dapp U, Anders J (2006) Aktive Gesundheitsförderung im Alter: Ein neuartiges Präventionsprogramm für Senioren, 2. Aufl. Kohlhammer, Stuttgart

26. Meier-Baumgartner HP, Anders J, Dapp U (2005) Präventive Hausbesuche. Gesundheitsberatung für ein erfolgreiches Altern. Vincentz, Hannover

27. Stuck AE, Kharicha K, Dapp U et al (2007) An international randomised controlled study of health risk appraisal for older persons based in general practice (ISRCTN28458424). BMC Med Res Methodol 7:2

28. Stuck AE, Kharicha K, Dapp U et al (2007) Development, feasibility and performance of a health risk appraisal questionnaire for older persons. BMC Med Res Methodol 7:1

29. Boult C, Dowd B, McCaffrey Det al (1993) Screening elders for risk of hospital admission. J Am Geriatr Soc 41:811-817

30. Stuck AE, Walthert J, Nikolaus T et al (1999) Risk factors for functional status decline in communitydwelling elderly people: a systematic literature review. Soc Sci Med 48:445-469

31. Dapp U, Anders J, von Renteln-Kruse W et al (2012) The longitudinal urban cohort ageing study (LUCAS): study protocol and participation in the first decade. BMC Geriatr 12:35 (http://www. biomedcentral.com/1471-2318/12/35). Zugegriffen: 1. Juli 2015

32. Dapp U, Lorentz Ch, Laub S et al (2009) Im Alter aktiv und gesund leben - Ergebnisse einer repräsentativen Seniorenbefragung in Hamburg. ZGerontol Geriat 42:245-255

33. Freie und Hansestadt Hamburg, Behörde für Gesundheit und Verbraucherschutz (2011) Die Gesundheit älterer Menschen in Hamburg II. Hamburg 2011. http://www.hamburg. de/gesundheitsberichte/3023506/berichtgesundheit-aelterer-menschen-2/. Zugegriffen: 15. April 2016

34. Dapp U, Jacobsen W, Fischer B (2013) „Aktiv und gesund leben in Eimsbüttel". Eine Befragung von Seniorinnen und Senioren im Bezirk Hamburg Eimsbüttel 2012 im Auftrag der Gesundheits- und Pflegekonferenz Hamburg Eimsbüttel. Abschlussbericht. http://www.hamburg.de/contentblob/ 4014430/data/eimsbuettel-pflegekonferenz bericht2012-teil-i-aktiv-und-gesund-leben-ineimsbuettel.pdf.Zugegriffen: 1. Juli 2015

35. McFadden D, Heiss F, Jun B et al (2006) On testing for independence in weighted contingency tables. Medium Econom Appl 14:11-18

36. Norton MC, Breitner JC, Welsh KA et al (1994) Characteristics of nonresponders in a community survey of the elderly. J Am Geriatr Soc 42:1252-1256

37. Bundeszentrale für politische Bildung, Statistisches Bundesamt, Wissenschaftszentrum Berlin für Sozialforschung, Sozio-oekonomisches Panel am Deutschen Institut für Wirtschaftsforschung (Hrsg) (2013) Datenreport 2013. Ein Sozialbericht für die Bundesrepublik Deutschland. Bundeszentrale für politische Bildung, Bonn

38. Motel-Klingebiel A, Wurm S, Tesch-Römer C (Hrsg) (2010) Altern im Wandel. Befunde des Deutschen Alterssurveys (DEAS). Kohlhammer, Stuttgart

39. Börsch-Supan $A$, Brugiavini $A$, Jürges $H$ et al (Hrsg) (2005) Health, Ageing and Retirement in Europe. First Results from the Survey of Health, Ageing and Retirement in Europe. Mannheim 
Research Institute for the Economics of Aging (MEA), Mannheim

40. Börsch-Supan A, Brugiavini A, Jürges H et al (Hrsg) (2008) First Results from the Survey of Health, Ageing and Retirement in Europe (2004-2007). Starting the Longitudinal Dimension. Mannheim Research Institute for the Economics of Aging (MEA), Mannheim

41. Brenner H(2011) DerForschungsverbund ESTHERnet. Interdisziplinäre Alternsforschung zur Multimorbidität und Gebrechlichkeit. Z Gerontol Geriat 44(Suppl 2):27-39

42. Peters A, Döring A, Ladwig KH et al (2011) Multimorbidität und erfolgreiches Altern. Ein Blick auf die Bevölkerung im Rahmen der KORA-AgeStudie.ZGerontol Geriat 44(Suppl 2):41-53

43. Saß AC, Wurm S, Ziese T (2009) Inanspruchnahmeverhalten. In: Böhm K, Tesch-Römer C, Ziese T (Hrsg) Beiträge zur Gesundheitsberichterstattung des Bundes: Gesundheit und Krankheit im Alter. Robert Koch-Institut, Berlin, S134-159

44. Hansen H,Schäferl, Schön Getal (2014) Agreement between self-reported and general practitionerreported chronic conditions among multimorbid patients in primary care - results of the MultiCare cohort study. BMC Fam Pract 5:39

45. Minder CE, Müller T, Gillmann G et al (2002) Subgroups of refusers in a disability prevention trial in older adults: baseline and follow-up analysis. Am J Public Health 92:445-450

46. Newman AB (2010) An overview of the design implementation, and analyses of longitudinal studies on aging. J Am Geriatr Soc 58(Suppl 2):S287-S291

47. Nationale Akademie der Wissenschaften Leopoldina, acatech - Deutsche Akademie der Technikwissenschaften, Union der deutschen Akademien der Wissenschaften (Hrsg) (2015) Medizinische Versorgung im Alter - Welche Evidenz brauchen wir? Nationale Akademie der Wissenschaften Leopoldina, Halle. https://www. leopoldina.org/uploads/tx_leopublication/2015_ 3Akad_Stellungnahme_Evidenzbasierung_web. pdf.Zugegriffen: 15. April 2016

48. German National Cohort (GNC) Consortium (2014) The German National Cohort: aims, study design and organization. Eur JEpidemiol 29:371-382

49. Kamtsiuris P, Lange M, Hoffmann R et al (2013) Die erste Welle der Studie zur Gesundheit Erwachsener in Deutschland (DEGS1). Stichprobendesign, Response, Gewichtung und Repräsentativität. Bundesgesundheitsbl Gesundheitsforsch Gesundheitsschutz 56:620-630

50. Statistisches Bundesamt (Hrsg) (2015) Bevölkerung und Erwerbstätigkeit 2014., Wiesbaden(https://www.destatis.de/DE/Publikationen/ Thematisch/Bevoelkerung/Bevoelkerungsstand/ VorlBevoelkerungsfortschreibung5124103149004. html). Zugegriffen: 1. Juli 2015

51. Behörde für Gesundheit und Verbraucherschutz (BGV) der Freien und Hansestadt Hamburg (2012) Eckpunkte für das Rahmenprogramm „Gesund alt werden in Hamburg!“ im „Pakt für Prävention Gemeinsam für ein gesundes Hamburg!". http:// www.hamburg.de/gesund-alt-werden/3585672/ eckpunktepapier-gaw2012/. Zugegriffen: 1. Juli 2015 